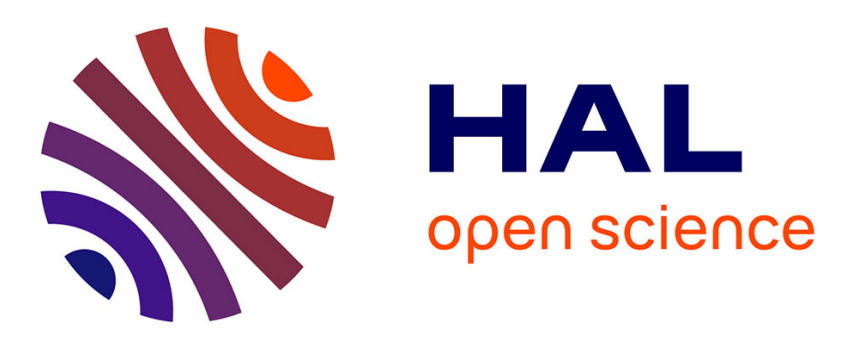

\title{
Ellipsoidal mesoscale mineralization pattern in human cortical bone revealed in 3D by plasma focused ion beam serial sectioning
}

Dakota Marie Binkley, Joseph Deering, Hui Yuan, Aurélien Gourrier, Kathryn Grandfield

\section{To cite this version:}

Dakota Marie Binkley, Joseph Deering, Hui Yuan, Aurélien Gourrier, Kathryn Grandfield. Ellipsoidal mesoscale mineralization pattern in human cortical bone revealed in 3D by plasma focused ion beam serial sectioning. Journal of Structural Biology, 2020, 212 (2), pp.107615. 10.1016/j.jsb.2020.107615 . hal-02967367

\section{HAL Id: hal-02967367 https://hal.science/hal-02967367}

Submitted on 14 Oct 2020

HAL is a multi-disciplinary open access archive for the deposit and dissemination of scientific research documents, whether they are published or not. The documents may come from teaching and research institutions in France or abroad, or from public or private research centers.
L'archive ouverte pluridisciplinaire HAL, est destinée au dépôt et à la diffusion de documents scientifiques de niveau recherche, publiés ou non, émanant des établissements d'enseignement et de recherche français ou étrangers, des laboratoires publics ou privés. 
Online version: https://www.sciencedirect.com/science/article/pii/S104784772030188X

doi: https://doi.org/10.1016/j.jsb.2020.107615

Please cite as: Binkley, D.M., Deering, J., Yuan, H., Gourrier, A., Grandfield, K. (2020). Ellipsoidal mesoscale mineralization pattern in human cortical bone revealed in $3 D$ by plasma focused ion beam serial sectioning, J. Struct. Biol. 212, 107615. doi: 10.1016/j.jsb.2020.107615

\title{
Ellipsoidal mesoscale mineralization pattern in human cortical bone revealed in 3D by plasma focused ion beam serial sectioning
}

\author{
Dakota Marie Binkley, ${ }^{\mathrm{a}, \mathrm{d}}$, Joseph Deering ${ }^{\mathrm{b}}$, Hui Yuan ${ }^{\mathrm{c}}$, Aurélien Gourrier ${ }^{\mathrm{d}, \mathrm{e}}$, Kathryn Grandfield ${ }^{\mathrm{a}, \mathrm{b} *}$ \\ a School of Biomedical Engineering, McMaster University, Hamilton, Canada \\ b Department of Materials Science and Engineering, McMaster University, Hamilton, Canada \\ c Canadian Centre for Electron Microscopy, McMaster University, Hamilton, Canada \\ d Univ. Grenoble Alpes, LIPHY, F-38000 Grenoble, France \\ e CNRS, LIPHY, F-38000 Grenoble, France \\ * Corresponding author: kgrandfield@mcmaster.ca
}

\begin{abstract}
Visualizing bone mineralization and collagen fibril organization at intermediate scales between the nanometer and the hundreds of microns range, is still an important challenge. Similarly, visualizing cellular components which locally affect the tissue structure requires a precision of a few tens of nanometers at maximum while spanning several tens of micrometers. In the last decade, gallium focused ion beam (FIB) equipped with a scanning electron microscope (SEM) proved to be an extremely valuable structural tool to meet those ends. In this study, we assess the capability of a recent plasma FIB-SEM technology which provides up to 50x increase in measurement speed over gallium FIB-SEM, thus paving the way to larger volume analysis. Nanometer-scale layers of demineralized and mineralized unstained human femoral lamellar bone were sequentially sectioned over volumes of $6-16,000 \mu \mathrm{m}^{3}$. Analysis of mineralized tissue revealed prolate ellipsoidal mineral clusters measuring approximately $1.1 \mu \mathrm{m}$ in length by $700 \mathrm{~nm}$ at their maximum diameter. Those features, suggested by others in high resolution studies, appear here as a ubiquitous motif in mineralized lamellar bone over thousands of microns cubed, suggesting a heterogeneous and yet regular pattern of mineral deposition past the single collagen fibril level. This large scale view retained sufficient resolution to visualize the collagen fibrils while also partly visualizing the lacuno-canalicular network in three-dimensions. These findings are strong evidence for suitability of PFIB as a bone analysis tool and the need to revisit bone mineralization over multi-length scales with mineralized tissue.
\end{abstract}

Keywords: Biomineralization; bone; FIB-SEM; PFIB; LCN; ultrastructure; serial sectioning; lamellar; cortical. 


\section{Introduction.}

Bone's remarkable mechanical properties are essentially determined by its macro-to-microarchitecture and tissue properties (Currey, 2002). Mineralized collagen fibrils with typical diameters of $100 \mathrm{~nm}$ are considered to be the fundamental building block of this multiscale structural hierarchy and tissue mechanics is primarily determined by fibril orientation and mineral density (Granke et al., 2013; Wagermaier et al., 2006; Weiner et al., 1999). Thus, the precise organization of collagen fibrils and of the mineral phase in healthy and pathological bone tissue is still an active area of research.

In addition to the intrinsic fibrillar organization pattern in a 1-10 $\mu \mathrm{m}$ range, resulting from tight biological control and self-assembly mechanisms, periodic modulations can be observed over distances up to $100 \mu \mathrm{m}$ in lamellar bone of many species. This characteristic length scale corresponds to the presence of vascular channels in human cortical bone around which the mineralized collagen fibrils are organized with high symmetrical regularity (Giraud-Guille, 1988; Wagermaier et al., 2006; Weiner et al., 1999). It also corresponds to the typical thickness of trabecular bone which is mostly found in the epiphyses of long bones, between the two surfaces of flat bones and also within irregular bones, such as vertebrae and the sacrum. This high degree of organization up to the microscopic scale is, however, partly perturbed by dendritic osteocytes embedded in the tissue, whose cell bodies are usually spaced apart by $\sim 25 \mu \mathrm{m}$ on average, depending on the tissue organization (Hannah et al., 2010; Kollmannsberger et al., 2017; Repp et al., 2017). This dense, highly interconnected cellular network is a key player for bone functions (Bonewald, 2011) and potentially constitutes a source of local tissue disorganization with respect to higher levels of symmetry. It is therefore becoming evident that analyzing the sub-microscopic structure of bone tissue also requires visualizing the lacuno-canalicular porosity network (LCN) hosting the osteocytes (Kerschnitzki et al., 2011) as well as the cellular details.

While a plethora of characterization tools exist for bone (Georgiadis et al., 2016) visualizing ultrastructural details with sufficient resolution to distinguish $100 \mathrm{~nm}$ diameter mineralized collagen fibrils while probing sufficiently large volumes with representative LCN organizations (in the $100 \mu \mathrm{m}$ range) is still an important technical challenge. A subset of ultrastructural characterization tools allows exploration of collagen-mineral arrangement with electron microscopy, chiefly transmission electron microscopy (TEM) as well as electron tomography (Grandfield et al., 2018; Landis et al., 1996; McNally et al., 2012; Reznikov et al., 2018; Weiner and Traub, 1992). These methods allow visualization of small volumes of up to $200 \mathrm{~nm}$ thick with sub-nanometer to angstrom resolution. Although TEM techniques have been central to defining collagen-mineral arrangement and mineral platelet morphology, even electron tomography is limited by geometrical restrictions and specimen thickness requirements, leading to missing wedge artifacts (Wang et al., 2016) which conflate reconstructions and therefore, some conclusions made via this method. Recently, another electron microscopy approach has surfaced as a highly complementary tool of TEM in bone hierarchical investigations, namely, focused ion beam (FIB) scanning electron microscopy (SEM), usually abbreviated to FIB-SEM, but also called FIB-SEM serial sectioning or FIB-SEM tomography. In this method, a heavy ion (in most cases from a liquid metal ion source, such as gallium $\left(\mathrm{Ga}^{+}\right)$) beam is used to sequentially mill material, the block-face of which is then imaged with a coincident electron beam. FIB-SEM enables visualization of larger volumes (approximately $10 \times 10 \times 10-20 \times$ $20 \times 20 \mu^{3}$ ) than TEM which partly compensates for the loss in resolution to a few nanometers. Indeed, FIBSEM serial-sectioning has been demonstrated on embedded or cryogenically preserved tissues including, mineralized turkey tendon (Zou et al., 2019), human, pig and rat lamellar bone (Reznikov et al., 2014a, 2014b, 2013), human trabecular bone (Reznikov et al., 2014a), mandibular bone of the minipig (Maria et al., 2019), zebrafish larvae (Akiva et al., 2019; Silvent et al., 2017), embryonic chicken bone (Kerschnitzki et al., 2016) and the interface of cementum and periodontal ligament (Hirashima et al., 2020b, 2020a) to name a few. Nearly all studies utilize demineralized bone and were therefore focused on the collagen fibrils. Very few studies capture large sections of the LCN, which were therefore performed with a much lower spatial resolution than technically possible, to acquire larger fields of view on mineralized bone tissue (Schneider et al., 2011). Therefore, so far, FIB-SEM studies of bone were designed to focus on either the collagen organization or the LCN and could not allow for investigation of both or the mineral within bone. 
A relatively new technology, plasma focused ion beam (PFIB-SEM) presents many advantages for capturing large scale volumes with high resolution. A PFIB is a dual beam FIB-SEM instrument that uses an inductively coupled Xenon $\left(\mathrm{Xe}^{+}\right)$plasma ion source. The more massive $\mathrm{Xe}+$ ion generally leads to higher material removal rates for the same ion beam current as $\mathrm{Ga}^{+}$-based FIB-SEM technology, for example material sputtering rates range from $\sim 10-30 \%$ to upwards of $\sim 300 \%$ higher for the same current and duration (Burnett et al., 2016). Conservative estimates therefore state that PFIB-SEM is $20-60 \mathrm{x}$ faster for $3 \mathrm{D}$ acquisition than $\mathrm{Ga}^{+}$-based FIBSEM (Altmann and Young, 2014; Burnett et al., 2016). Not only the ion source, but also the ion optics of the two systems vary. The PFIB is capable of much higher ion beam currents (few pA to $>1 \mu \mathrm{A}$ ) compared to Ga-FIB (1pA to 20nA), and retains a focused spot size unlike $\mathrm{Ga}+$ at high ion beam currents to enable thin slicing (Smith et al., 2006). The PFIB also maintains a quality surface for imaging, with the amount of surface amorphization decreased by $20-40 \%$ on silicon compared to $\mathrm{Ga}^{+}-\mathrm{FIB}$ (Kelley et al., 2013), and the surface roughness reduced by an order of magnitude (from 400 to $50 \mathrm{~nm}$ RMS on interconnects) when using a rocking polish (Altmann and Young, 2014). These potential benefits translate for biological imaging in $3 \mathrm{D}$, ultimately offering possibility for larger, smoother, and less damaged volumes to be acquired by the PFIB in shorter times with the same nanoscale resolution as images afforded by the $\mathrm{Ga}^{+}$FIB-SEM (Bassim et al., 2014; Burnett et al., 2016). As outlined in Figure 1, this 3D imaging allows a portion the osteocyte lacuno-canalicular network and the tissue to be probed simultaneously. To our knowledge, PFIB-SEM has yet to be employed in the investigation of bone ultrastructure to microscale structure (also referred to as mesoscale structure), despite its success in characterizing other materials with porous features, including concrete (Burnett et al., 2016) and hydroxyapatite coatings on biomedical alloys (Hu et al., 2017).

In this work, we apply PFIB-SEM serial sectioning to human bone for the first time. We highlight substantial contrast differences between demineralized and mineralized human femoral lamellar bone following widely reported staining and embedding sample preparation protocols. We show that, without having to go through the burdensome procedure of demineralizing and staining, similar or better imaging performances can be achieved on mineralized bone. This offers new perspectives which we explore to probe the mesoscale organization of mineralized collagen fibrils in human bone. We highlight the presence of ellipsoidal mineral clusters, a potentially underestimated intermediate level of organization between collagen fibrils and lamellae in human osteonal bone. We also show that the extent of the probed volume allows visualization of a large portion of the $\mathrm{LCN}$ while retaining sufficient spatial resolution to observe collagen features.

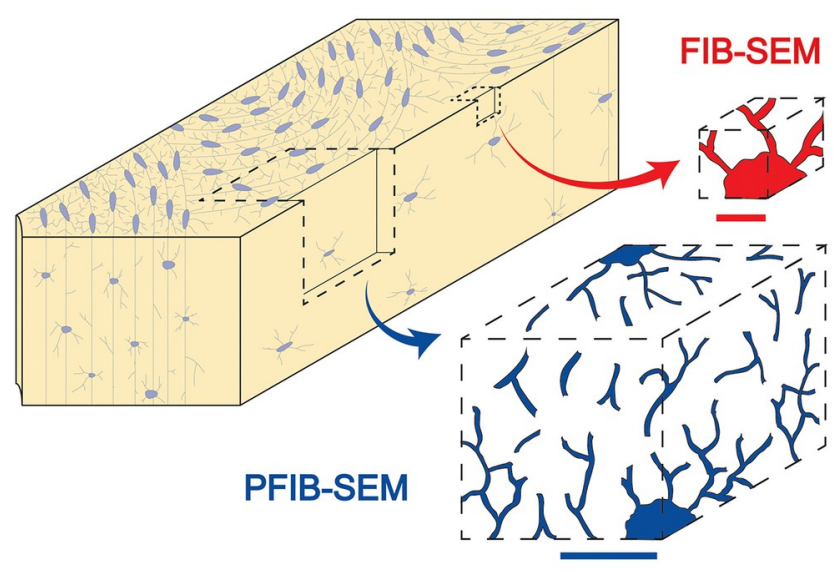

Figure 1. Comparative accessible bone volumes with FIB vs PFIB-SEM with respect to LCN features. Size restrictions in conventional FIB-SEM techniques (red) essentially allow imaging a single osteocyte lacuna in bone tissue due to their inherently small volumes. The PFIB-SEM technique (blue) is able to probe volumes that are an order of magnitude larger than conventional FIB-SEM methods, allowing for high-resolution 3D reconstruction of a larger proportion of lacunae and canaliculi, thus theoretically providing a broader visualization of the LCN network characteristics. Red scale bar: $10 \mu \mathrm{m}$. Blue scale bar: $50 \mu \mathrm{m}$. 


\section{Materials and methods.}

Specimen: A fresh-frozen human femur of a 68-year-old male, with no known bone disease, was obtained with institutional ethical approval (HIREB No. 12-085-T) and immediately upon thawing, fixed in a $4 \%$ glutaraldehyde (Sigma Aldrich, Missouri, US) solution in a $0.1 \mathrm{M}$ cacodylate buffer for 7 days. The bone was sectioned using a slow speed diamond saw (Buehler Isomet, Illinois, US) under hydrated conditions. A longitudinal section, approximately $2 \mathrm{~mm}$ thick, was cut along the length of the femur for demineralization. It was imaged normal to the cutting plane. A transverse section, also $2 \mathrm{~mm}$ thick, was extracted for immediate dehydration and embedding, remaining in its naturally mineralized state. This section was imaged normal to the cutting plane. A schematic detailing sample orientation is shown in Figure S1.

Demineralization and Staining Protocols: In preparation for demineralization, the longitudinally sectioned bone was polished to approximately $200 \mu \mathrm{m}$ in thickness with 400, 800, 1200, and 2400 grit emery paper and finally a $50 \mathrm{~nm}$ diamond suspension on a polishing cloth (Buehler, Illinois, US). The sample was demineralized by immersion in a solution of 5\% ethylenediaminetetraacetic acid (EDTA) (Sigma Aldrich, Missouri, US) and $2 \%$ paraformaldehyde (PFA; Sigma Aldrich, Missouri, US) in cacodylate buffer, $\mathrm{pH} 7$ until the sample was visibly transparent (approximately $72 \mathrm{hrs)}$ ). The demineralized bone was rinsed 10 times with deionized water prior to staining. The sample was then pre-stained with Alcian blue using a modified version of a previously published protocol (Reznikov et al., 2014b). The sample was placed in 5\% Alcian blue in an aqueous solution was heated to $30^{\circ} \mathrm{C}$ and cooled to room temperature on a hot plate for a total of 10 cycles to increase the uptake of the dye and decrease aggregation. The demineralized bone was rinsed 5 times with deionized water prior to subsequent staining. The sample was then stained by successive treatments with osmium tetroxide and thiocarbohydrazide (OTOTO) according to previously described methods (Reznikov et al., 2013).

Mineralized Sample Preparation: Unlike the demineralized sample, the mineralized sample was neither demineralized nor stained. The fixed $2 \mathrm{~mm}$ thick transverse section directly underwent the dehydration and embedding protocol below.

Dehydration and Embedding Protocols for Demineralized and Mineralized Samples: Both the demineralized and mineralized tissue were dehydrated in a graded series of ethanol $(70 \%, 80 \%, 90 \%, 95 \%$, $100 \%$ ) for 12 hours each, and further dehydrated in 100\% propylene oxide (Sigma Aldrich, Missouri, US). The tissues were gradually infiltrated $(25 \%, 50 \%, 75 \%, 100 \%)$ with EMbed 812 resin (Electron Microscopy Sciences, USA) in propylene oxide (Sigma Aldrich, Missouri, USA). The embedded blocks were then cured in an oven at $60^{\circ} \mathrm{C}$. The top surface and adjacent cross-section of the embedded bone were polished with 400, 800, 1200, and 2400 grit emery paper, and a $50 \mathrm{~nm}$ diamond suspension on a polishing cloth (Buehler, Illinois, US) to expose the bone in the resin.

Sample Coating: The polished demineralized and mineralized bone was placed on standard SEM stubs using silver paint. Both the exposed top face and cross-section intended for PFIB-SEM slicing were coated with $5 \mathrm{~nm}$ of gold using a Precision Etching Coating System (PECS II) coater (Gatan Inc., CA, USA) to minimize charging effects.

PFIB-SEM Serial Sectioning: Demineralized Tissue A Xenon-sourced PFIB microscope (Helios G4 UXe, Thermo Scientific, Hillsboro, USA) equipped with a Schottky field-emission gun SEM was employed. To protect the region of interest, a $63 \times 47 \times 5 \mathrm{~m}^{3} \mathrm{Pt}$ capping layer was deposited on the top surface using a $20 \mathrm{nA}$ and $12 \mathrm{keV}$ ion beam. Brief capping layer experiments (see supplementary methods, Table S1 and S2 and Figure S2) were completed to determine suitable protective layer compositions to minimize curtaining artifacts attributed to ion channeling and preferential milling. Suitable protective layer compositions for demineralized and mineralized tissues were qualitatively determined to be platinum $(\mathrm{Pt})$ and carbon $(\mathrm{C})$, respectively.

Trenches adjacent to the area of interest were milled in order to expose the cross-section and reduce shadowing to detectors and the re-deposition of material. An X-shaped fiducial was milled into the exposed cross-section to allow for post-processing data alignment though translational registration. The electron beam was focused on the exposed cross-section at $2 \mathrm{keV}$ and $1.6 \mathrm{nA}$ under 'immersion mode', with a working distance of $5.9 \mathrm{~mm}$, pixel 
width of $20 \mathrm{~nm}$, a $500 \mathrm{~ns}$ dwell time with 2 frame integrations. Imaging was completed with a retractable concentric backscattered detector (CBS). Sequential milling and images were collected using automated Slice and View software (Thermo Scientific, OR, USA), with a $4 \mathrm{nA}$ ion current, $30 \mathrm{keV}$ accelerating voltage, $20 \mathrm{~nm}$ slice thickness, and a $4^{\circ}$ stage rocking angle, which has been demonstrated to minimize curtaining artifacts (Loeber et al., 2017). A full workflow is visualized in Figure S3. The final cropped volume of the demineralized bone was $45.8 \times 40.9 \times 8.7 \mu \mathrm{m}^{3}$.

PFIB-SEM Serial Sectioning: Mineralized Tissue Similarly to the demineralized dataset, a protective capping layer $50 \mu \mathrm{m} \times 50 \mu \mathrm{m} \times 8 \mu \mathrm{m}$ of $\mathrm{C}$ was deposited on the area of interest using ion beam deposition at $60 \mathrm{nA}$ and $12 \mathrm{keV}$. An X-shaped fiducial was milled into the exposed cross-section to allow for post-processing data alignment. The electron beam was focused on the exposed cross-section at $1.5 \mathrm{keV}$ and $0.8 \mathrm{nA}$, with a working distance of $2.7 \mathrm{~mm}$, pixel width of $12.5 \mathrm{~nm}$, a $500 \mathrm{~ns}$ dwell time, with 8 frame integrations. Imaging was completed with an in-lens detector under backscatter mode. Tomography data was collected using automated Slice and View software (Thermo Scientific, OR, USA), with $1 \mathrm{nA}$ ion current, $30 \mathrm{keV}$ accelerating voltage, 25 $\mathrm{nm}$ slice thickness, and a $7^{\circ}$ stage rocking angle. The final cropped tomogram of the mineralized bone was 28.6 $\mu \mathrm{m} \times 25.6 \mu \mathrm{m} \times 8.7 \mu \mathrm{m}$.

PFIB Data Reconstruction and Visualization: The tomographic datasets were processed using Dragonfly 4.1 (Objects Research Systems, QC, Canada). Datasets were aligned using a cross-correlation approach and curtain artifacts were removed using the image processing toolbox available in Dragonfly. Shadowing effects from the SEM image aligning fiducial were removed on the demineralized images by changing the shading compensation, applying a histogram balance, and also by applying a contrast limited adaptive histogram equalization to the mineralized images. The images were reconstructed, and the osteocyte network was segmented using a U-Net classifier trained with 15 manually segmented images. Segmentations were inspected against the original datasets and minor corrections were implemented manually, including some denoising and selection of missed canaliculi on 2D slices. The machine learning outputs and revised datasets were transformed to a thickness mesh to calculate the canalicular diameter (Figure S4). Cellular organelles and the cell body were segmented manually. Small volumes were extracted from the mineralized dataset to view the mineral morphology, where a Gaussian filter $(\sigma=0.5)$ was applied to reduce noise. Orthogonal slices, two-dimensional, and three-dimensional volumes were extracted from these smaller datasets.

It is important to note some geometrical information and the assignment of planes and directions. In our volumes, the XY plane always represents the milling and image plane during acquisition while the $\mathrm{YZ}$ and $\mathrm{XZ}$ planes are reconstructed after slice registration. By this convention, $\mathrm{Z}$ is always the thickness of the serial sectioning dataset. A clarification of the sample geometries from the bulk tissue are clarified in Figure S1.

\section{Results and discussion.}

\section{PFIB on Mineralized versus Demineralized Bone Tissue}

PFIB-SEM was conducted on both demineralized and mineralized bone. A section of demineralized and stained lamellar bone was reconstructed into a volume measuring $45.8 \times 40.9 \times 8.7 \mu^{3}$ with an isotropic voxel size of $20 \mathrm{~nm}$, while a slightly smaller 3D volume measuring $28.6 \times 25.6 \times 8.7 \mu \mathrm{m}^{3}$ with an anisotropic voxel size of $12.5 \times 12.5 \times 25 \mathrm{~nm}$ was obtained on the mineralized sample. A side-by-side comparison of similar regions, neighboring an osteocyte lacuna, and measuring the same size of approximately $6 \mu \mathrm{m} \times 7 \mu \mathrm{m}$ is shown in Figure 2. What is immediately apparent between the two specimens is the drastic change in contrast when mineral is present. This contrast must logically be associated to mineralization fluctuations in the tissue. Upon closer inspection of the mineralized data, this contrast takes the form of regular bright patches scattered throughout the imaged volume surrounded by a darker border with dimensions in the micron range. In the demineralized sample, only slight, ill-defined change in contrast is detected which could be due to preferential staining of regions previously mineralized, or to remnant mineral still causing electron beam scattering. 

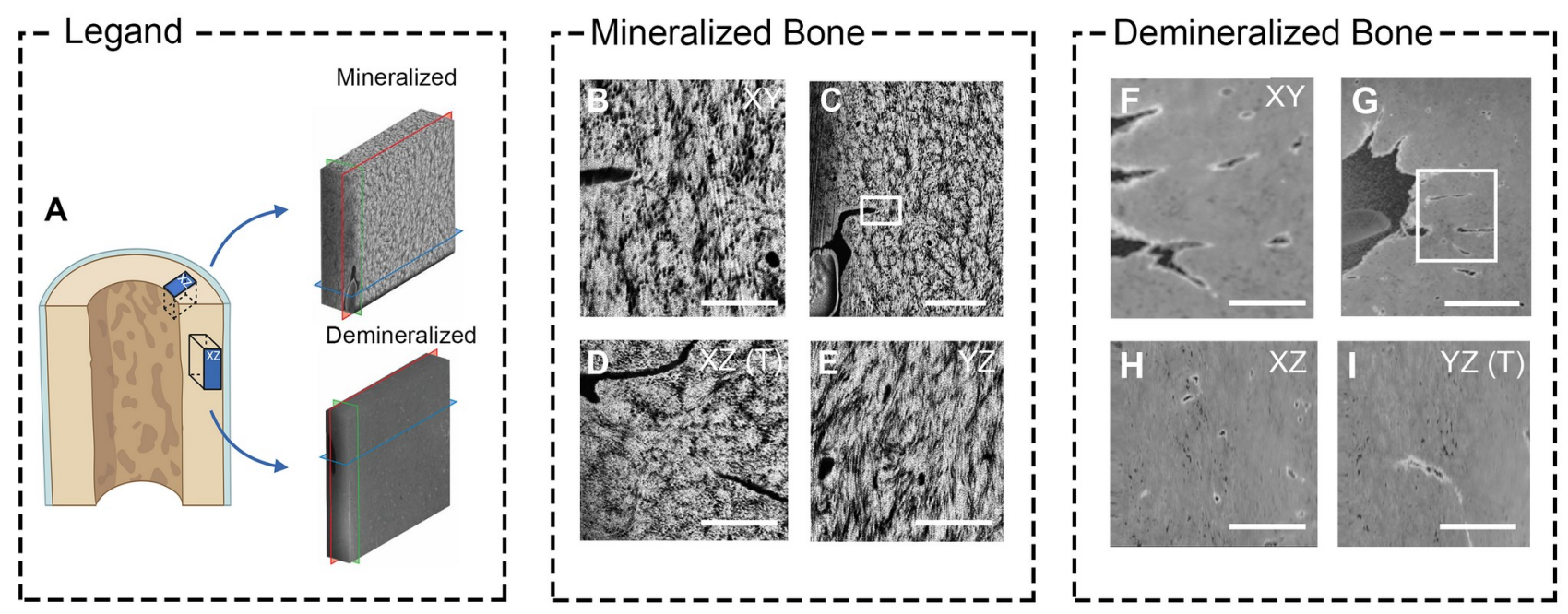

Figure 2. Comparison of mineralized and demineralized bone analyzed by FIB-SEM. (A) A schematic demonstrating that the mineralized and demineralized samples were sectioned in the transverse and longitudinal directions, respectively. Sub-volumes between the mineralized and demineralized bone were selected in regions near the osteocyte lacuna (B,D) and canaliculi $(\mathrm{C}, \mathrm{E})$ for comparison along orthogonal planes. In the mineralized sample, marquises are evident on the (B) XY and (G) YZ planes, while rosettes are revealed in the (F) XZ plane, which is the anatomically transverse section of the bone, denoted by "(T)". In the demineralized sample, very faint outlines of marquise-like shapes are noted in the $(\mathrm{H}) \mathrm{XZ}$ plane and faint rosettes on (I) the YZ plane, which corresponds to the anatomically transverse section of the bone. Scale bars: (B,D) $5 \mu \mathrm{m}$, remainder $2 \mu \mathrm{m}$.

Figure A was created using BioRender.com.

Both preparation methods enabled the detection of canaliculi, apparent as circular or linear black voids (mineralized sample), or black voids with a brightly stained periphery (demineralized stained sample). While FIB-SEM serial sectioning has been widely used on a combination of demineralized and mineralized bone tissue from a variety of species (a summary of previous studies is presented in Table 1), these works have focused on investigating other aspects of human bone than mineral. For example, a mineralized volume of murine bone focused on elucidating the LCN network connections (Schneider et al., 2011), while a mineralized and heavily stained sample of chick embryonic calvaria showed the collagen arrangement during ossification (Hashimoto et al., 2017). Mineralized zebrafish larvae were also investigated (Silvent et al., 2017) and showed pathways for ion transport through blood vessels (Akiva et al., 2019), but no similar mineral clusters to size and shape of those noted herein were highlighted. A major finding of this work is therefore the ability to visualize previously unnoticed distinct mineral clusters by FIB-SEM within human cortical bone when using mineralized and unstained sections of bone.

\section{Evidence of ubiquitous ellipsoidal microscopic mineral clusters in bone tissue}

Closer inspection of the full mineralized data in 3D and along orthogonal directions is shown in Figure 3. The mineral clusters appear more circular in the transverse direction (Figure 3D) and more elongated longitudinally (Figure 3B). To analyze the mineral clusters, five sub-volumes (cubes with side length of $3 \mu \mathrm{m}$ ) were selected along a random diagonal direction (Figure 4 and Figure S4). These highlight more clearly the shape and orientation of those mineral clusters: they appear more circular in the $\mathrm{XZ}$ plane (transverse to osteon), irrespective of the ROI location in the sample and are more elongated in the orthogonal planes, especially YZ where the smallest features have dimensions compatible with mineralized collagen fibrils aligned in the longitudinal plane. Extracting one representative mineral cluster and showing all its orthogonal planes and as a $3 \mathrm{D}$ reconstruction (Figure 5), the shape of these structures in 3D more closely resembles a prolate ellipsoid, than a perfect sphere, the elongation direction being the osteonal axis. Several more of these clusters were segmented (Figure S5) and dimensions extracted, measuring on average $700 \pm 164.4 \mathrm{~nm}$ in diameter at their maximum midpoint and $1.14 \pm 0.26 \mu \mathrm{m}$ in length (Table S3). The circular-like shape is shown across the whole XZ plane in Supplementary Movie 1 and the true ellipsoidal nature of these in more detail in Supplementary Movie 2. 
Table 1. Partial summary of FIB-SEM serial sectioning on various mineralized tissues. When not available, slice thickness $(\mathrm{Z})$ is given in place of voxel size, and total thickness of tomogram (ZT) is given in place of all tomogram dimensions in $\mathrm{X}, \mathrm{Y}, \mathrm{Z}$.

\begin{tabular}{|c|c|c|c|c|c|}
\hline $\begin{array}{c}\text { Tissue } \\
\text { Human Bone }\end{array}$ & Preparation & Tomogram Size & Voxel Size & Volume $\left(u^{3}{ }^{3}\right)$ & Reference \\
\hline Cortical bone & Demineralized & $45.8 \times 40.9 \times 8.7 \mu \mathrm{m}$ & $20 \times 20 \times 20 \mathrm{~nm}$ & 16297 & Present work \\
\hline Cortical bone & Mineralized & $28.6 \times 25.6 \times 8.7 \mu \mathrm{m}$ & $12.5 \times 12.5 \times 25 \mathrm{~nm}$ & 6370 & Present work \\
\hline Cortical bone & Demineralized & $\mathrm{N} / \mathrm{A}$ & $10 \times 10 \times 10 \mathrm{~nm}$ & $\mathrm{~N} / \mathrm{A}$ & (Reznikov et al., 2018) \\
\hline Cortical bone & Demineralized & $Z_{T}=6-9 \mu \mathrm{m}$ & $\begin{array}{l}10 \times 10 \times 10 \mathrm{~nm} \text { to } \\
12.5 \times 2.5 \times 12.5 \mathrm{~nm}\end{array}$ & $\mathrm{~N} / \mathrm{A}$ & (Reznikov et al., 2014b) \\
\hline $\begin{array}{l}\text { Trabecular } \\
\text { bone }\end{array}$ & Demineralized & $Z_{T}=6-9 \mu m$ & $\begin{array}{l}10 \times 10 \times 10 \mathrm{~nm} \text { to } \\
12.5 \times 12.5 \times 12.5 \mathrm{~nm}\end{array}$ & $\mathrm{~N} / \mathrm{A}$ & (Reznikov et al., 2014a) \\
\hline \multicolumn{6}{|c|}{ Other Mammalian Bone } \\
\hline $\begin{array}{l}\text { Minipig alveolar } \\
\text { bone }\end{array}$ & Demineralized & $Z_{T}=10-15 \mu \mathrm{m}$ & $\begin{array}{l}10 \times 10 \times 10 \mathrm{~nm} \text { to } \\
12.5 \times 12.5 \times 12.5 \mathrm{~nm}\end{array}$ & $\mathrm{~N} / \mathrm{A}$ & (Maria et al., 2019) \\
\hline $\begin{array}{l}\text { Minipig } \\
\text { fibrolamellar } \\
\text { bone }\end{array}$ & Demineralized & $\begin{array}{c}X=10.24 \mu \mathrm{m} \\
Y=8.6 \mu \mathrm{m}\end{array}$ & $10 \times 10 \times 10 \mathrm{~nm}$ & N/A & (Magal et al., 2014) \\
\hline $\begin{array}{l}\text { Murine } \\
\text { trabecular bone }\end{array}$ & Demineralized & $25 \times 20 \times 20 \mu \mathrm{m}$ & $Z=50 \mathrm{~nm}$ & 10000 & (Hasegawa et al., 2018) \\
\hline $\begin{array}{l}\text { Murine proximal } \\
\text { tibial } \\
\text { metaphysis }\end{array}$ & Demineralized & $40 \times 40 \times 40 \mu \mathrm{m}$ & $20 \times 20 \times 20 \mathrm{~nm}$ & 64000 & (Robles et al., 2018) \\
\hline Murine femur & Mineralized & $19 \times 14 \times 11 \mu \mathrm{m}$ & $18 \times 18 \times 29.5 \mathrm{~nm}$ & 2926 & (Schneider et al., 2011) \\
\hline $\begin{array}{l}\text { Murine bone- } \\
\text { osteoblastic } \\
\text { processes }\end{array}$ & Demineralized & $30 \times 20 \times 17.5 \mu \mathrm{m}$ & $Z=50 \mathrm{~nm}$ & 10500 & (Hasegawa et al., 2017) \\
\hline $\begin{array}{l}\text { Rat lamellar } \\
\text { bone }\end{array}$ & Demineralized & $10 \times 10 \times 2-10 \mu \mathrm{m}$ & $\mathrm{Z}=10 \mathrm{~nm}$ & $200-1000$ & (Reznikov et al., 2013) \\
\hline \multicolumn{6}{|c|}{ Embryonic Avian Bone } \\
\hline Long bones & Demineralized & N/A & N/A & N/A & (Kerschnitzki et al., 2016) \\
\hline Calvaria bone & Mineralized & $25 \times 25 \times 25 \mu \mathrm{m}$ & $25 \times 25 \times 25 \mathrm{~nm}$ & 15625 & (Hashimoto et al., 2017) \\
\hline \multicolumn{6}{|l|}{ Tendon } \\
\hline Turkey & $\begin{array}{l}\text { Mineralized } \\
\text { Demineralized }\end{array}$ & N/A & $\begin{array}{l}12 \times 12 \times 24 \mathrm{~nm} \\
6 \times 6 \times 8.7 \mathrm{~nm}\end{array}$ & N/A & (Zou et al., 2019) \\
\hline Rat & Demineralized & $Z_{T}=260 \mu \mathrm{m}$ & $Z=60 \mathrm{~nm}$ & $\mathrm{~N} / \mathrm{A}$ & (Kanazawa et al., 2014) \\
\hline $\begin{array}{l}\text { Dental Materials } \\
\text { Mice cementum }\end{array}$ & Demineralized & $73 \times 63 u \mathrm{~m} \times 8 \mu \mathrm{m}$ & $36 \times 36 \times 100 \mathrm{~nm}$ & 36792 & (Hirashima et al., 2020b) \\
\hline Rat dentin & Demineralized & $73 \times 63.6 \times 4.2 \mu \mathrm{m}$ & $36 \times 36 \times 100 \mathrm{~nm}$ & 19500 & (Tanoue et al., 2018) \\
\hline $\begin{array}{l}\text { Zebrafish } \\
\text { Zebrafish larvae }\end{array}$ & $\begin{array}{l}\text { Demineralized } \\
\text { and mineralized }\end{array}$ & $26 \times 22 \times 12 u m$ & $26 \times 26 \times 26 \mathrm{~nm}$ & 6864 & (Silvent et al., 2017) \\
\hline Zebrafish larvae & $\begin{array}{l}\text { Mineralized, } \\
\text { cryo-FIB-SEM }\end{array}$ & $\begin{array}{c}30 \times 23 \times 12 u m \\
40 \times 23 \times 24.5 u m\end{array}$ & $\begin{array}{l}10 \times 10 \times 20 \mathrm{~nm} \\
20 \times 20 \times 40 \mathrm{~nm}\end{array}$ & $\begin{array}{l}8280 \\
22540\end{array}$ & (Akiva et al., 2019) \\
\hline
\end{tabular}


Similar mineral clusters have been identified on the floor of osteocyte lacunae (Shah et al., 2016) and more recently, at the apex of rat calvarial sutures (Shah et al., 2020) by exposing bone to deproteinization protocols. In Shah et al. the shape has been identified as a marquise, which is not uniform throughout bone, but rather changes to a less organized marquise moving away from the bone mineralization front, suggesting that this shape is a transient early form of mineral deposit (Shah et al., 2020). The same general shapes, identified as prolate ellipsoids, have been shown in the turkey tendon (Zou et al., 2019), and over small sections of human bone, e.g. Movie S1 in Reznikov et al. (Reznikov et al., 2018).
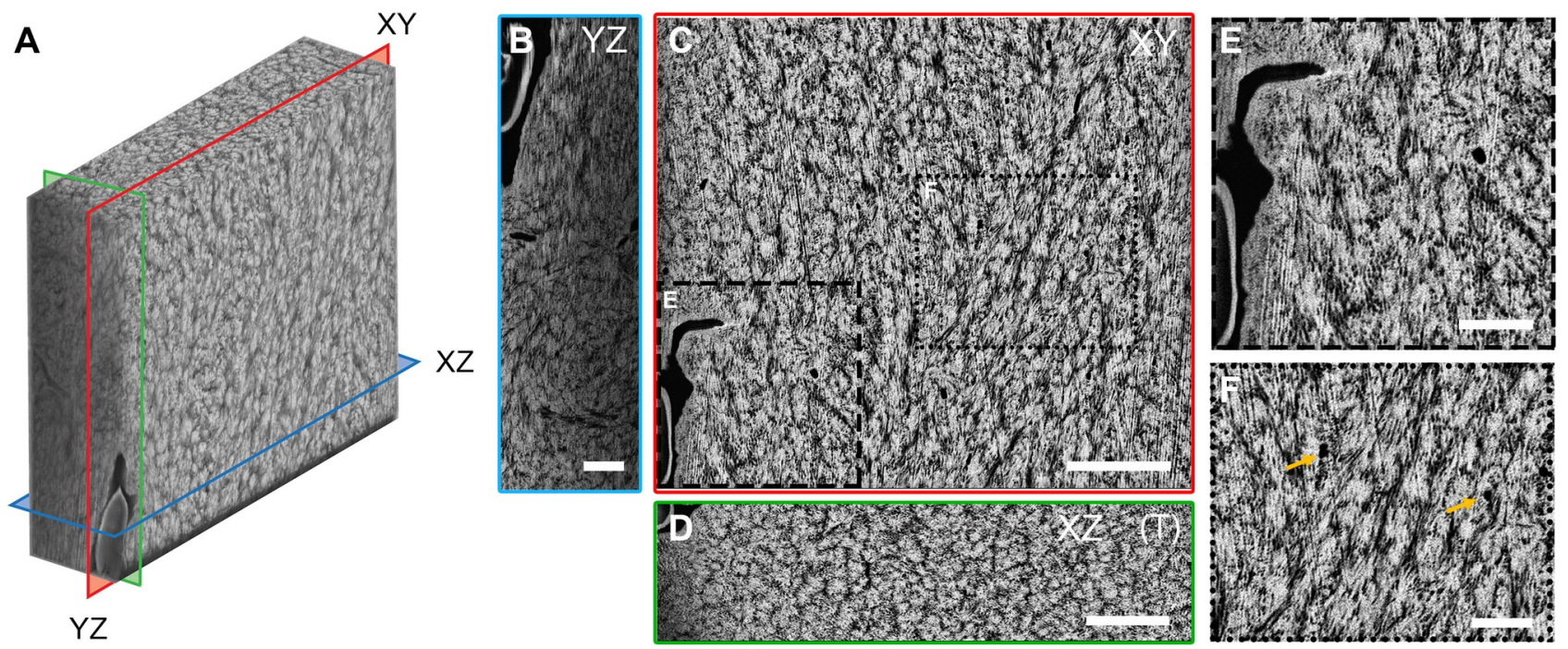

Figure 3. PFIB-SEM of mineralized lamellar bone. (A) 3D reconstruction of mineralized bone with an isotropic voxel size of $25 \mathrm{~nm}$ and total volume measuring $28.6 \mu \mathrm{m}$ x $25.6 \mu \mathrm{m} \times 8.7 \mu \mathrm{m}$. YZ (green), XY (red), and XZ (blue) orthogonal planes are annotated to show the location of the images in B, C, and D, respectively. Mineral appears light grey/white, while canalicular networks, porosity and collagen all share black contrast. (B) An image extracted from the YZ plane of the reconstructed volume, note an osteocyte in the top left corner and dark canaliculi traversing the view on oblique angles. Scale bar: $2 \mu \mathrm{m}$. (C) An image of the XY plane. In this view, the mineral component of bone appears bright, while canaliculi appear as black round circles (inset F). An osteocyte is present in the bottom left (inset E). Scale bar: $5 \mu \mathrm{m}$. (D) An image extracted from the XZ plane of the reconstructed volume, corresponding to the transverse anatomical plane. Scale bar: $3 \mu \mathrm{m}$. (E) Inset of (C), with osteocyte (bottom left) showing a clear membrane (light grey) and the LCN, which appears black. Cell processes were not visible within canaliculi extending from this osteocyte. Mineral appears finer near the canaliculi periphery, but may be due to shadowing. Scale bar: $2 \mu \mathrm{m}$. (F) Inset of (C), where white arrows point to circular canaliculi. Note that due to the absence of staining, canaliculi in this case appear black. Scale bar: $2 \mu \mathrm{m}$.

We note that this structure is ubiquitous throughout an entire $45 \mu \mathrm{m}$ section of lamellar bone and represents a hierarchical level of mineral organization above the single collagen fibril level of $100 \mathrm{~nm}$ in diameter and below the typical dimensions of an osteon lamella of 5-7 $\mu \mathrm{m}$. It also shows that this structure is not a member of the "disorganized" sub-lamella motif (Reznikov et al., 2013) associated with canaliculi. The two earlier papers citing ellipsoidal mineral shapes have also postulated that the presence of a cross-collagen fibril mineralization motif may be possible (Reznikov et al., 2018; Zou et al., 2019). Our findings on the microscale, depicting mineral clusters that span roughly $700 \mathrm{~nm}$ in width and just over $1 \mu \mathrm{m}$ in length across over a distance of $45 \mu \mathrm{m}$ clearly indicate that mineral is indeed associated with more than one collagen fibril, confirming earlier hypotheses that tissue mineralization occurs in patterns regularly spaced over large distances past the single collagen fibril level. 


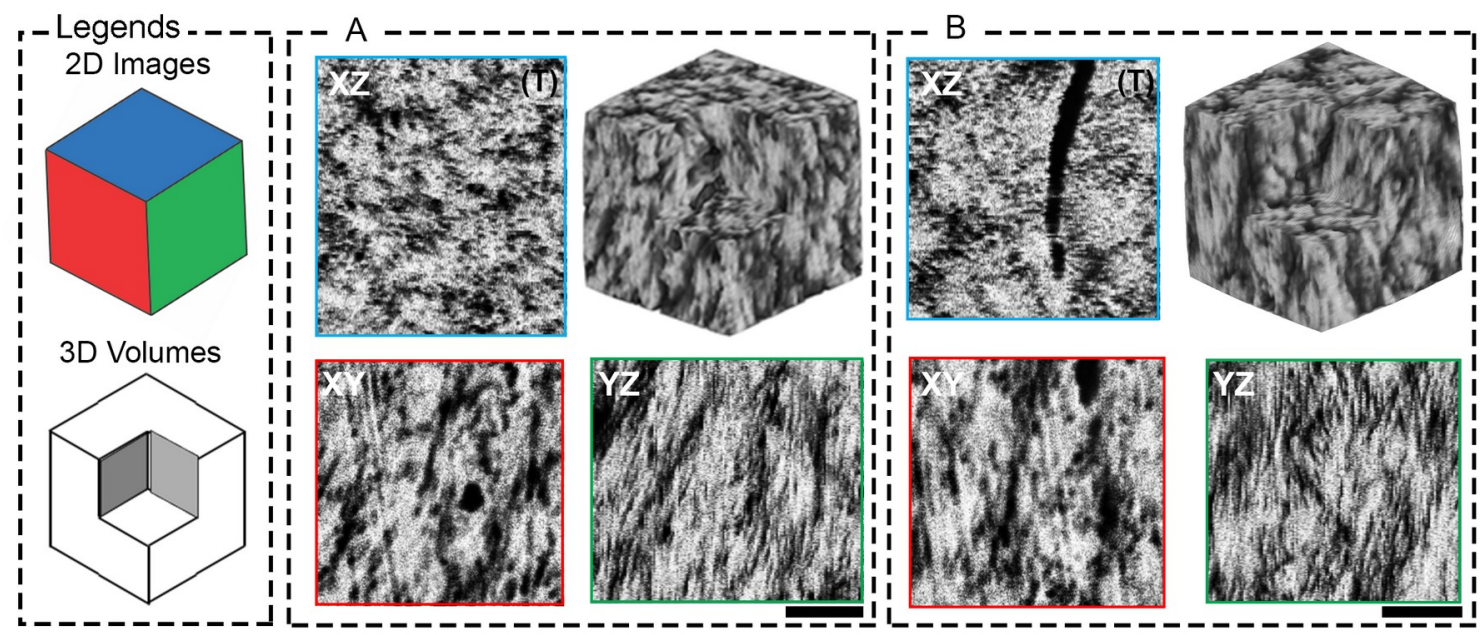

Figure 4. Exploring mineral and collagen orientation in 3D The PFIB volume is cropped into smaller volumes $(\mathrm{A}, \mathrm{B})$ measuring $3 \mu \mathrm{m}$ each side to explore the orientation of mineral and collagen fibrils. The transverse planes (XZ) show clear rosette shapes, while the YZ plane along the long axis of the femur, shows collagen fibrils in plane, denoted by their characteristic banding pattern. XY planes show the faint outline of diamond, or marquise shapes.
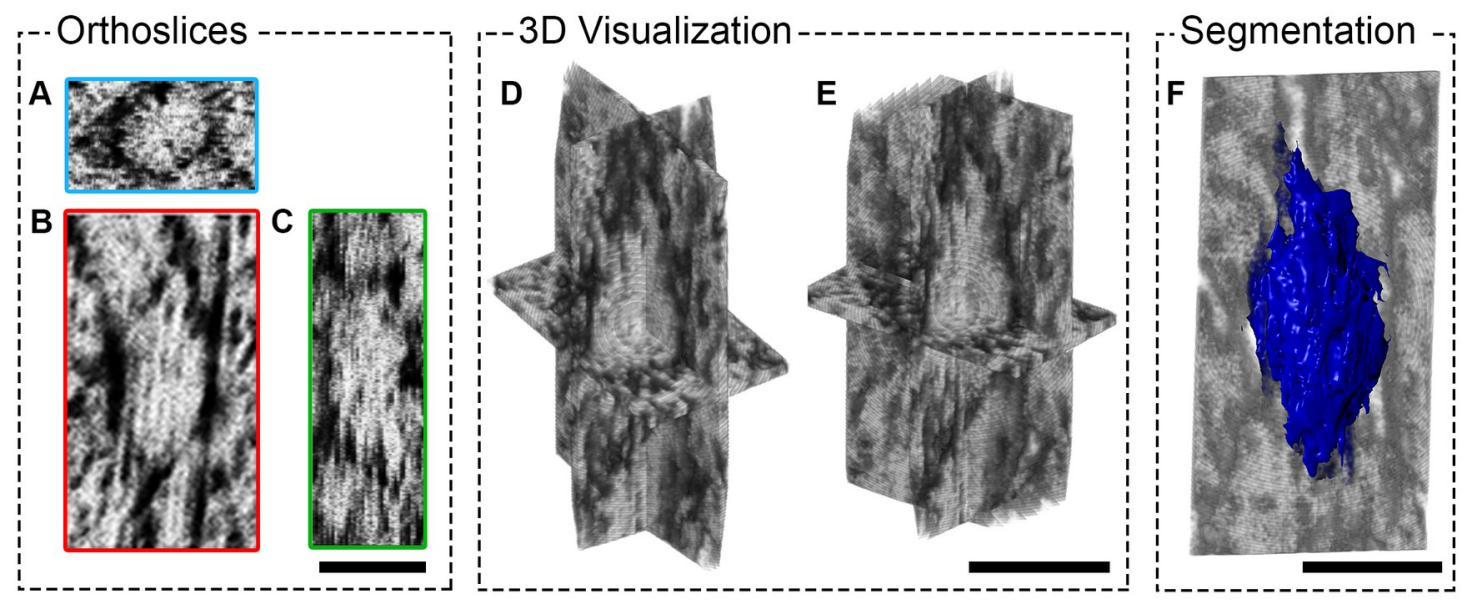

Figure 5. Mineral cluster morphology in 3D. A representative mineral cluster from PFIB-SEM of the mineralized sample has been isolated to probe its shape both by separated $(A, B, C)$ and intersecting orthogonal planes $(D, E)$. In (A) the transverse slice, perpendicular to the long axis of the femur, is given by plane XZ. Here, a clear rosette shape is visible also discernible from the top-down tilted view in (E). Segmentation of the 3D shape in (F) confirms its likely shape as a prolate ellipsoid. All scale bars: $1 \mu \mathrm{m}$.

Seminal works focusing on early bone formation by Bonucci et al. and Bernard and Pease outline similar spherical shapes as "calcification loci" or "round bodies" between collagen fibrils in osteoid (Bernard and Pease, 1969; Bonucci, 1971). These are thought to elongate, firstly unrelated to collagen banding, then lastly become associated with the fibril interior, or to coarsen from calcification loci to bone nodules which coalesce to form all mineralized tissue (Bernard and Pease, 1969; Bonucci, 1971). These findings are supported by cryo electron microscopy studies, which observe the accumulation of mineral on the exterior of the fibril in in vitro conditions (Nudelman et al., 2013). Similar spherical particles 'calcospherulites' have been postulated to be related to the mineralization front only (Midura et al., 2008) and early bone mineralization from in vitro culture of MC3T3-E1 osteoblast cells show comparable spherical particles (mineralization foci), and also notes these in mouse calvarial bone with osteopontin closely associated with their margin (Addison et al., 2014). It remains to be determined if the ellipsoidal shapes uncovered herein once began as spherical clusters, as so predominantly noted in early bone formation literature. 


\section{The mineral: linking to the nanoscale}

Viewing the mineral clusters in the transverse (XZ) plane, means that we view a section perpendicular to the long axis of the osteon, closely aligned along the femur axis in our case and, presumably, of the collagen fibrils. In such an orientation the circular shape is fully consistent with previously observed features in high-resolution TEM (Figure 6) in human bone, termed "rosettes" (Grandfield et al., 2018) , "lacy" pattern (Reznikov et al., 2018) or otherwise unnamed in the alveolar bone of minipigs (Maria et al., 2019). Dispersed between some rosettes are clear black circular or linear voids marking the canaliculi, and a substantial portion of black volume surrounds each rosette. Upon closer inspection of Figure 6B, a unique feature of each rosette is its mineral-dense center (Supplemental Movie S3).

The rosette features noted by (Grandfield et al., 2018) in large TEM sections, are reproduced in higher resolution images in Figure 6C and D for comparison. With the higher resolution and compositional contrast afforded by high angle annular dark field scanning transmission electron microscopy (HADDF STEM), we begin to make sense of the black regions surrounding the rosettes as viewed in PFIB-SEM. The features are outside of the resolution limit of the PFIB-SEM, but with the STEM images, we can see individual mineral plates exist in this space, and are clearly resolved, wrapping around perfectly circular black volumes. Since these black regions correspond to low density regions in the SEM, we postulate that these are one of two features; sub-cellular or sub-micron level porosity within the extracellular matrix itself or collagen viewed in cross-section, or possibly a combination of both.
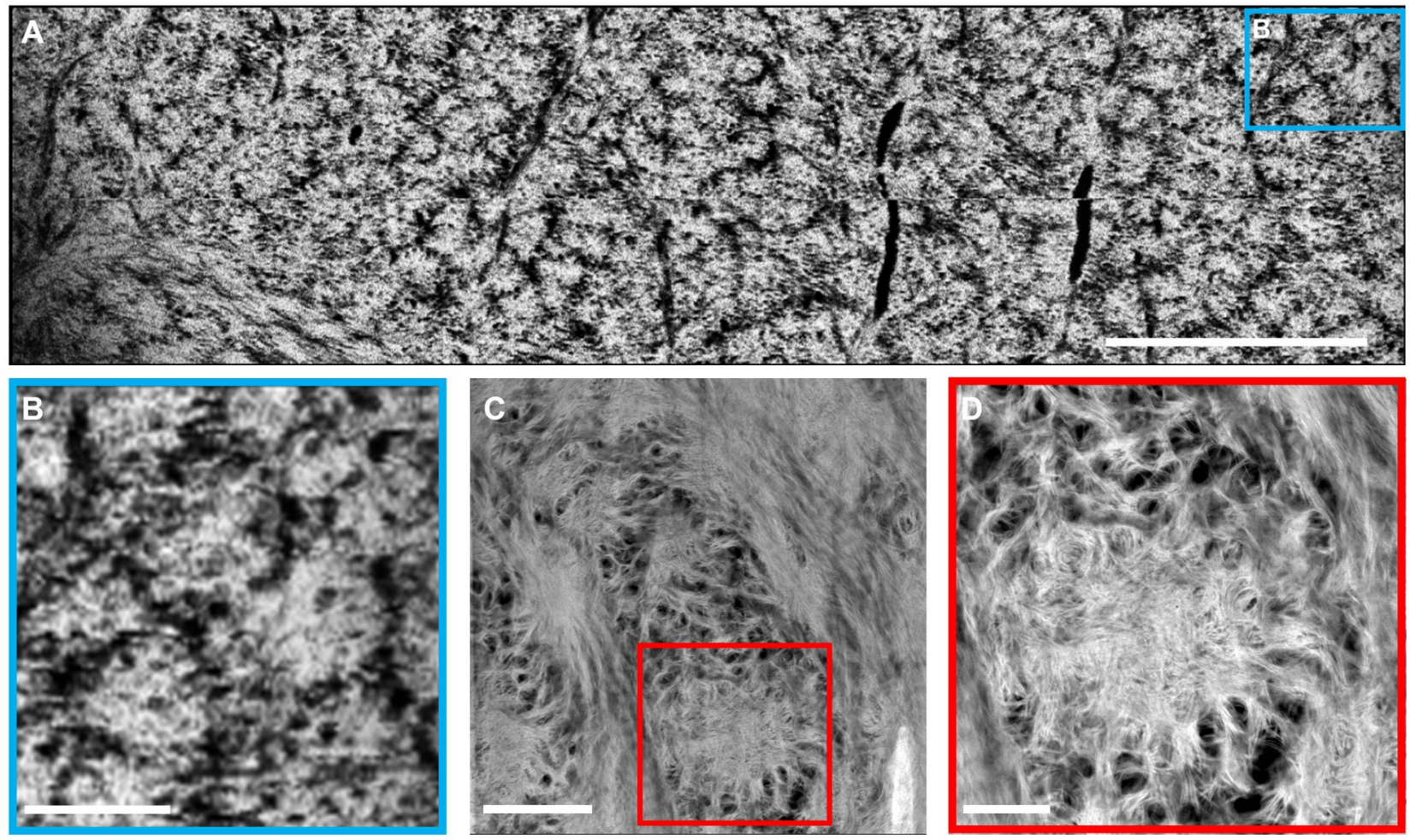

Figure 6. Mineral clusters linked to the nanoscale. A transverse slice (perpendicular to the long axis of the femur) from the mineralized PFIB-SEM volume is shown in (A) with higher resolution inset in (B). Scale bars: $5 \mu \mathrm{m}$ and 1 $\mu \mathrm{m}$, respectively. The mineral appears in rosette shapes across the entirety of the section. Black space surrounds the clusters, or takes the form of black lines represent the canalicular network running through the section. A lacuna is located near the bottom left corner, but not shown on this slice. A side-by-side comparison with STEM-HAADF images of human femoral bone (C) with higher magnification inset (D), reprinted with permission from (Grandfield et al., 2018), shows that the black space in PFIB surrounding the rosettes is comprised of mineral plates, wrapping around circular volumes, likely representing collagen, while the central portion remains densely mineralized. Scale bars: $500 \mathrm{~nm}$ and $200 \mathrm{~nm}$, respectively. 
While many comprehensive reviews cover the hierarchical levels of bone mineralization (Reznikov et al., 2014c; Weiner and Traub, 1992), we should draw attention to some of the competing views on collagen-mineral arrangement, chief of which is the concept of intra versus interfibrillar mineralization, referring to mineral either occupying (mainly the gap zone of) the collagen fibril, or external to the fibril/between fibrils. This conflicting view essentially stems from convincing results reported over decades supporting both observations. For example, X-ray or neutron diffraction peaks can readily be observed at small angles with the same $67 \mathrm{~nm}$ periodicity as this exhibited by non-mineralized collagen fibrils of bone or tendon, pointing to intrafibrillar mineralization in the collagen fibril gap zone (Bigi et al., 1988), as confirmed by TEM (Weiner and Traub, 1989). More recent STEM results obtained with FIB-SEM ultrathin samples suggest, on the contrary, that mineral nanoparticles are quasi-exclusively extrafibrillar (McNally et al., 2012; Schwarcz, 2015). Recent STEM tomography suggests a motif of cross-fibrillar mineralization, both intra-and interfibrillar (Reznikov et al., 2018). However, Landis et al. clearly reported electron microscopy evidence for the presence of both intra and extrafibrillar mineral in turkey tendon more than three decades ago (Landis et al., 1991) which is likely confirmed by our STEM observations of rosettes with a potential mix of inter and intrafibrillar mineralization.

Nevertheless, how this occurs or what this looks like remains elusive. Our images over large volumes with 12.5 $\mathrm{nm}$ resolution in $\mathrm{X}$ and $\mathrm{Y}$ and $25 \mathrm{~nm}$ resolution in $\mathrm{Z}$, complemented by those from Grandfield et al. (Grandfield et al., 2018), suggest the potential for a combination of intra and interfibrillar mineralization. Since the black circular regions on the periphery of the mineral cluster make up such a large fraction of the sample, it is highly unlikely they are all vacant of any material and therefore may not only represent sub-micron porosity. Hence, at least a part likely represent collagen fibrils in cross-section and the bright mineral encircling them therefore, represents interfibrillar mineralization between each collagen fibril. Collagen, as a light element, will not scatter electrons well in the PFIB-SEM or TEM when unstained as in the case of the mineralized sample herein, meaning that it likely will always appear black in cross-section. The presence of collagen in these cross-sectional dark regions has been confirmed in TEM sections using elemental analysis (Lee et al., 2019). On the other hand, the central area of the rosettes, viewed in our PFIB and the complementary STEM (Figure 6 B,C,D), suggests that the central portion may be much more densely infiltrated with mineral. A close up slice-by-slice view of one mineral cluster is highlighted in Movie S3, where the bright mineral is concentrated in the central region and more sparsely on the periphery. Yet, even in these densely mineralized regions, there are still signs of discrete particles albeit less well defined than at the rosette periphery. While PFIB-SEM is still in its infancy, optimization of the voxel size and slice thickness will enable probing this hypothesis in the future. Indeed, with the addition of elemental information by energy dispersive X-ray spectroscopy (EDX) mineral transport in other species has been shown on single slices of a block-face (Kerschnitzki et al., 2016b). Multi-spectral tomography or serial sectioning, i.e. collection of both images and EDX spectra slice-by-slice, could confirm both nanoscale structures and mineral locations in the future.

\section{PFIB-SEM for the visualization of the LCN}

One other potential advantage of PFIB-SEM serial sectioning is the large volumes quickly accessible with high resolution which enable exploration of the osteocyte and the LCN. Here we begin by assessing demineralized and stained bone, the usual sample preparation method for FIB-SEM. The demineralized reconstructed section is shown alongside three orthogonal planes (Figure 7B,C,D), where key features are the osteocyte in the top-left and its surrounding lacuna and canalicular extensions. While a boundary between the osteocyte and the lacuna is indistinguishable, perhaps due to a lack of high pressure freezing sample preparation, some distinct membranebound cellular organelles were present and segmented of which, the nucleus is most prominent (Figure 7E). In all slices, the canaliculi are clearly resolvable, primarily as relatively circular features with a bright boundary, due to osmium tetroxide staining, in the XY plane (arrows Figure 7F) or as oblong, branch-like features in YZ and XZ (Figure 7B, D). While a separate slice in Figure 2G highlights the capability to image the distinct collagen banding patterns also with PFIB-SEM which is a hallmark of most FIB-SEM serial sectioning performed on demineralized bone focused on fibril orientation (Reznikov et al., 2014b, 2013). 

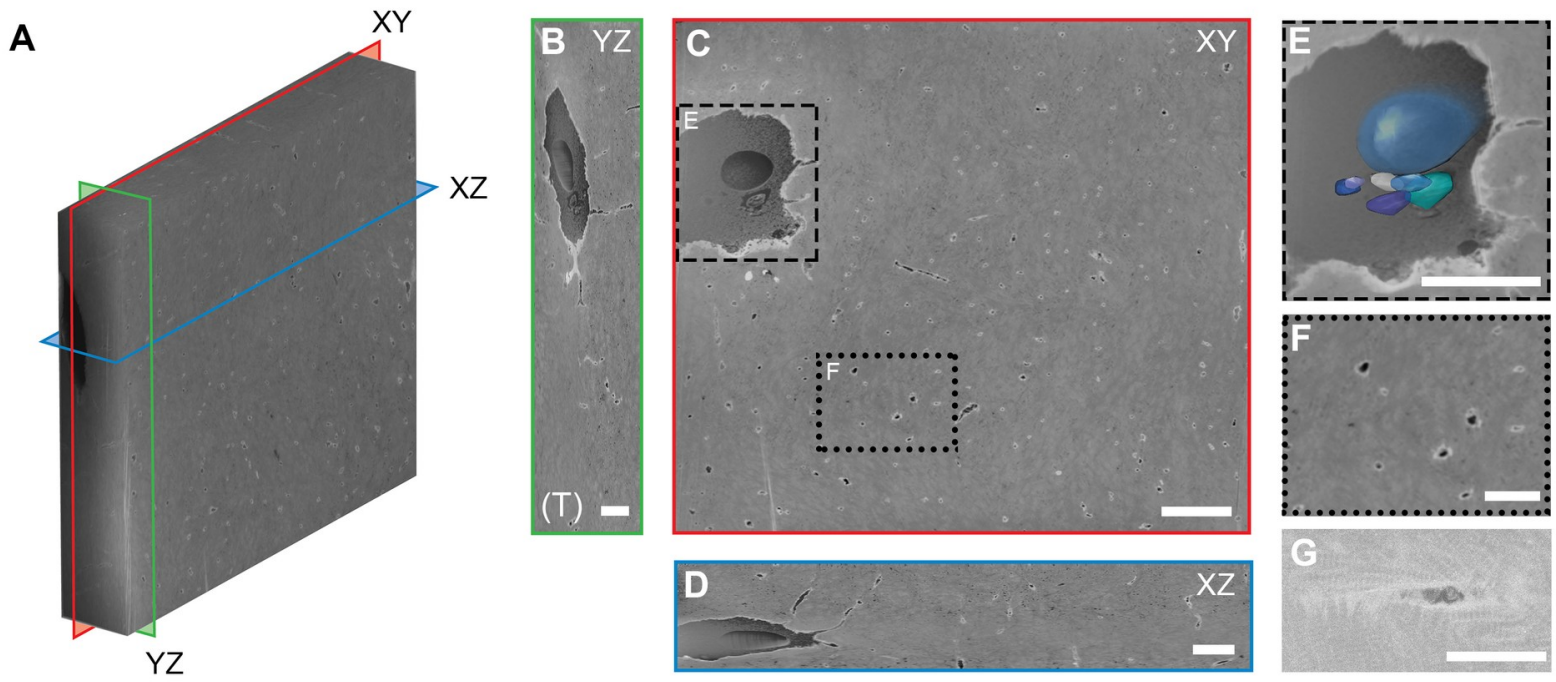

Figure 7. PFIB-SEM of demineralized lamellar bone (A) 3D reconstruction of demineralized bone with an isotropic voxel size of $20 \mathrm{~nm}$ and total volume measuring $45.8 \mu \mathrm{m}$ x $40.9 \mu \mathrm{m} \times 8.7 \mu \mathrm{m}$. YZ (green), XY (red), and XZ (blue) orthogonal planes are annotated to show the location of the images in B, C, and D, respectively. (B) An image extracted from the YZ plane of the reconstructed volume, with osteocyte visible. Scale bar: $2 \mu \mathrm{m}$. (C) An image of the $\mathrm{XY}$ plane. In this view, canaliculi appear as bright circles (inset F). An osteocyte is resolved within the dataset (inset E). Scale bar: $5 \mu \mathrm{m}$. (D) An image extracted from the XZ plane of the reconstructed volume. Scale bar: $3 \mu \mathrm{m}$. (E) Inset of (C), showing the osteocyte within its lacuna with higher magnification, segmented cellular organelles are overlaid on the image. Scale bar: $5 \mu \mathrm{m}$. (F) Inset of (C), where white arrows point to canaliculi in cross-section. Due to staining, their circular periphery appears bright white. Scale bar: $2 \mu \mathrm{m}$. $(\mathrm{G})$ A higher magnification image from another demineralized area, showing characteristic collagen banding around a canalicula. Scale bar: $1 \mu \mathrm{m}$.

The segmentation of the LCN from this demineralized dataset is shown in Figure 8A and B, with segmented cellular organelles in $\mathrm{C}$ and D. Here, the canaliculi appear to extend outwards from the osteocyte lacuna in all directions, with many connections also observed extending up from the bottom right-hand side of Figure 8A. Indeed, another osteocyte was located just outside of the volume captured in the tomogram, but visualized during set-up (shown in Figure S6). Therefore, the center of the volume approximately represents the meeting zone of the canaliculi from at least two osteocytes. The canalicular diameter in this segmentation was $346 \pm 146$ $\mathrm{nm}$ (Figure $8 \mathrm{E}$ ). The volume and segmented LCN of the demineralized sample are available in Supplementary Movie 4.

A slightly smaller 3D volume of the mineralized set is shown in Figure 3A. Similarly, the reconstructed volume is shown alongside its orthogonal planes (Figure 3B,C,D), where the XY plane was acquired during imaging, and the $\mathrm{YZ}$ and $\mathrm{XZ}$ planes created after image registration. While PFIB can mill extremely large volumes, the precise control of slice thickness well-resolved features in all planes. By avoiding laborious demineralization and staining processes, the resultant volumes and cross-sections show much different contrast. The bright white to light-grey contrast is representative of heavy matter, in this case the mineral, while any empty space, canaliculus, or light elements, like collagen, appear black. An osteocyte with its outer membrane in its lacuna is visible in the bottom left of the section (Figure 3E), although cellular processes are not. These cellular features have been well characterized using traditional FIB-SEM of osteocytes in trabecular bone (Hasegawa et al., 2018, 2017) and near atomic resolution using TEM tomography (Kamioka et al., 2012). Canaliculi are visible as black circles (arrows Figure $3 \mathrm{~F}$ ) in the $\mathrm{XY}$ plane and appear as oblong shapes or tubular features (Figure 3B, D) that span the entirety of the $\mathrm{YZ}$ and $\mathrm{XZ}$ plane. 

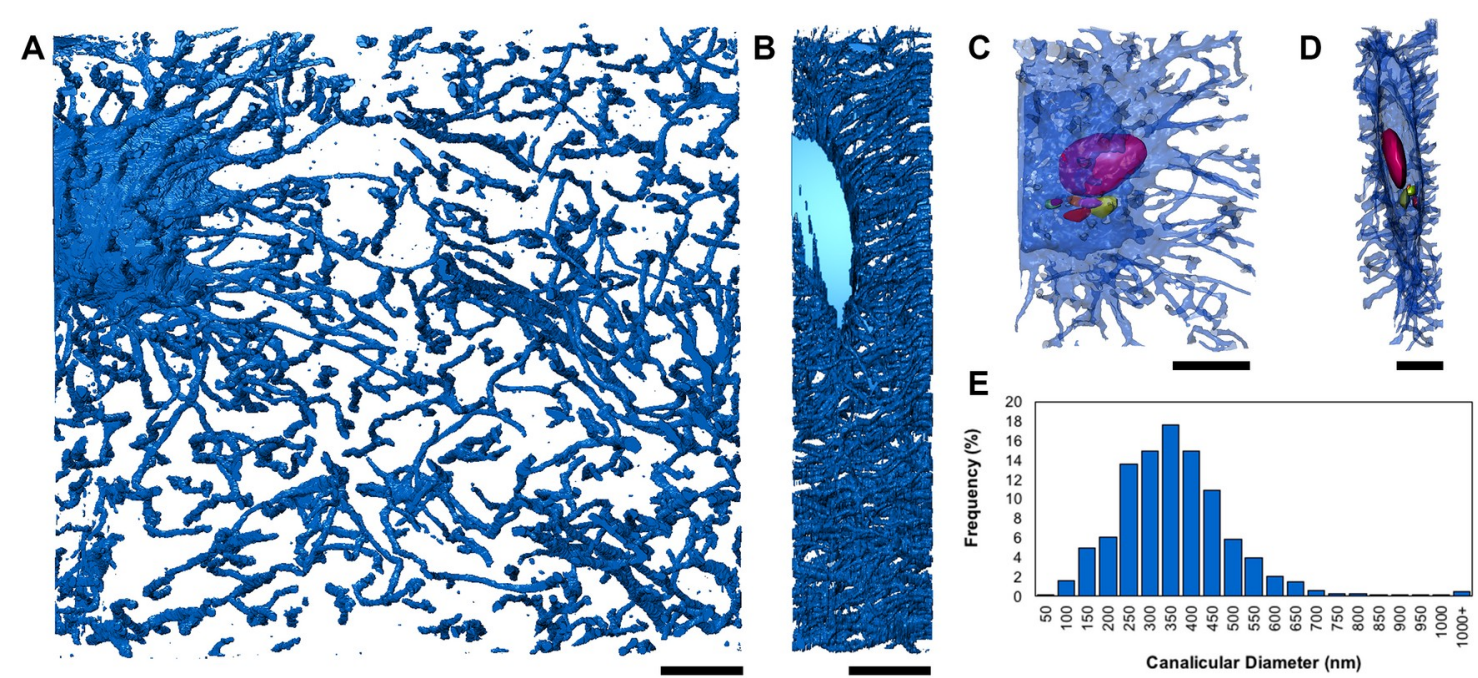

Figure 8. LCN network in demineralized lamellar bone. (A) XY projection of the segmented LCN where canalicular extensions span the volume. More canaliculi appear near the osteocyte cell in the top left corner, and near the bottom right corner of the volume, which is confirmed to be proximal to a second osteocyte that was not captured

in the PFIB-SEM dataset (Figure S5). Scale bar: $10 \mu \mathrm{m}$. (B) The segmented LCN from the YZ projection, which shows connections spanning the depth of the reconstruction. Scale bar: $5 \mu \mathrm{m}$. (C) A segmentation of the osteocyte and nearby extending canaliculi with resolvable cellular organelles segmented (multi-coloured) in 3D. Scale bar: $5 \mu \mathrm{m}$. (D) The cell from the YZ plane showing the 3D distribution of cellular organelles. Scale bar: $3 \mu \mathrm{m}$. (E) A frequency histogram of canalicular diameter from the segmentation. The average canalicular diameter is $346 \pm 146 \mathrm{~nm}$.
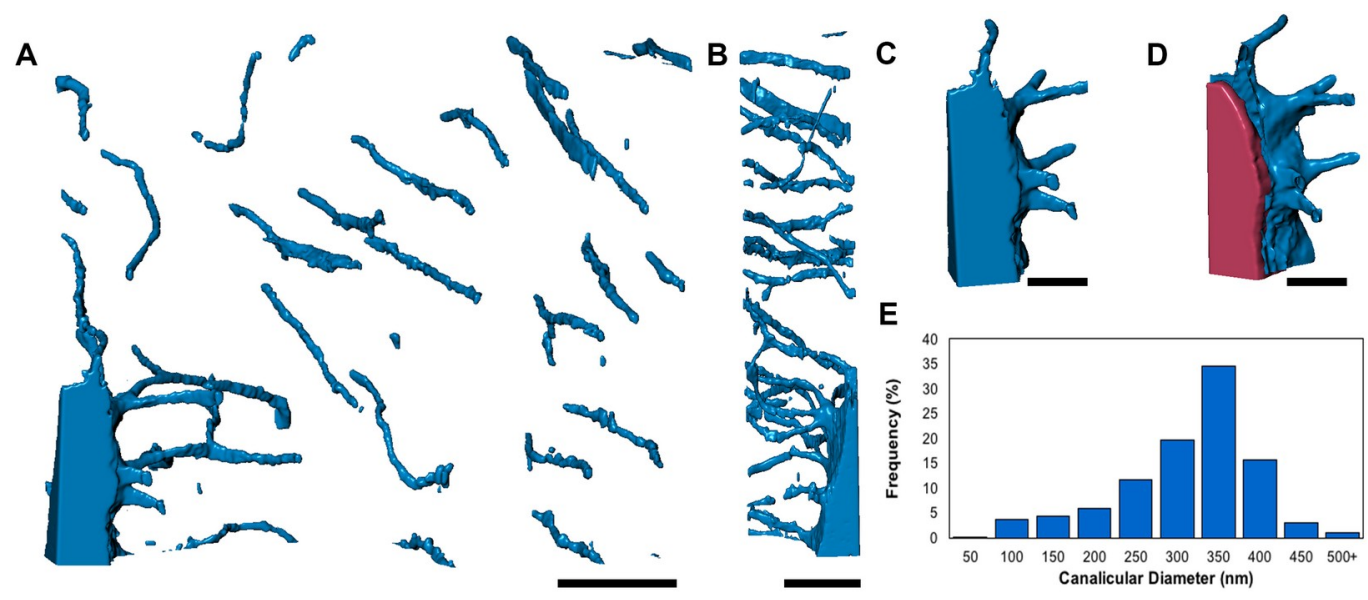

Figure 9. LCN network in mineralized lamellar bone (A) XY projection of the segmented LCN where long canalicular connections appear to extend primarily along the Z-direction of the reconstruction. This corresponds to along the $\mathrm{XZ}$ or transverse plane, therefore likely part of a network that would span osteonal layers. Part of an osteocyte is present in the bottom left corner of the segmentation. Scale bar: $5 \mu \mathrm{m}$. (B) The segmented LCN from the YZ view, which shows co-aligned connections spanning the depth of the reconstruction, with most being aligned in this direction. Scale bar: $5 \mu \mathrm{m}$. (C) A segmentation of the lacunae and extending space that (D) the osteocyte cell (pink) resides in. Scale bar $2 \mu \mathrm{m}$. (E) A frequency histogram of canalicular diameter from the segmentation. The average canalicular diameter is $285 \pm 82 \mathrm{~nm}$.

The segmentation of the LCN from this mineralized dataset is shown in Figure 9A and B, with what appears to be the cellular membrane, and not the lightly scattering osteopontin-rich hypocalcified lamina limitans that also exists around osteocytes (McKee and Nanci, 1996), segmented in C and D. However, we do note that for investigation of cellular structures, different sample preparation methods and analysis, including cryo-FIB would be more suitable. Most of the canaliculi appear co-aligned in the Z-direction (Figure 9B) within this volume, with minimal branching across the volume. It is well known the canalicular density can vary strongly within 
different regions of a bone sample. Also, in osteons, osteocytes are flattened in the tangential plane (in the lamellar plane) with the highest density of canaliculi oriented radially. Recall, that the samples were cut in orthogonal directions and planes were assigned such that the XY plane was the milling and image plane in PFIBSEM (Figure S1). Based on the images of segmented canaliculi, it is most likely that the demineralized XY plane falls radially with respect to an osteon, while the mineralized XY plane likely falls tangentially with respect to an osteon. The average canalicular diameter in the mineralized bone was $284 \pm 82 \mathrm{~nm}$ (Figure 9E), still in line with values reported by other techniques but a narrower range in comparison to the diameters reported for the demineralized bone above $(346 \pm 146 \mathrm{~nm})$. This difference could either be explained by a loss of mineral at the canalicular surface during demineralization, or to the difficulty to precisely define this interface due to a relative diffuse staining at the canaliculi surface of demineralized bone. Interestingly, $33 \%$ of the values fall in a relatively narrow range of 300-400 $\mathrm{nm}$ for the demineralized sample while $58 \%$ of the canaliculi diameters are slightly more broadly distributed within $250-450 \mathrm{~nm}$. For the mineralized sample, $50 \%$ are between $300-400 \mathrm{~nm}$ and $73 \%$ between $250-450 \mathrm{~nm}$. It is important to note, however, that a precise quantification of canaliculi diameter strongly depends on the segmentation procedure. Figure S7 highlights differences in segmentation, when meticulously corrected manually (shown in Figure 8 and 9), or when segmentation is completely relaxed and automated. The volume and segmented LCN of the mineralized sample are available in Supplementary Movie S5.

Osteocytes or LCN have been studied with a variety of techniques. Functional studies mostly rely on optical microscopy, due to the large variety of available fluorescent dyes that allow targeting specific cellular components and biomarkers (Ciani et al., 2009; Sano et al., 2015). Confocal and non-linear two-and threephoton microscopy have also been used to study the LCN characteristics and have the additional advantage of allowing large fields of views (Genthial et al., 2017; Kerschnitzki et al., 2011; Repp et al., 2017). However, all these methods suffer the classical diffraction limitation and the spatial resolution is typically limited to $\sim 200 \mathrm{~nm}$ laterally and $\sim 500 \mathrm{~nm}$ axially, such that the smallest canaliculi are out of reach, thus complicating network connectivity analysis. Higher resolution afforded by SEM was used to report canaliculi diameters in a range of $259 \pm 129 \mathrm{~nm}$ on 2D sections of mice humeri (You et al., 2004). Similarly, atomic force microscopy (AFM) studies report canaliculi diameters of $426 \pm 118 \mathrm{~nm}$ on 2D sections of cortical bovine tibia (Lin and Xu, 2010).

To overcome the limits of dimensional quantification on 2D observations, pseudo-3D visualization is often performed with SEM using resin-embedded and acid-etched bone replica (Bonewald, 2011; Shah and Palmquist, 2017). In 3D, two partial osteocyte lacunae and their junctions in murine bone were probed by traditional FIBSEM (Schneider et al., 2011). However, the roughly $20 \mu \mathrm{m}$ wide view did not capture a full lacuna, nor an osteocyte maintained within. While osteocytes and individual organelles were not classified in this work, as was not the aim, the feasibility to probe cellular organelle structures opens a potential avenue for further PFIB-SEM applications.

$\mathrm{X}$-ray tomography is also frequently used to assess various characteristics of the LCN. However, reaching sufficient resolutions to capture the smallest canaliculi still remains a challenge. Canaliculi with diameters of $320-390 \pm 120 \mathrm{~nm}$ were reported for human cortical femurs analyzed by X-ray phase contrast synchrotron nanotomography (SR-nanoCT) (Varga et al., 2015), but such measurements currently provide typical resolutions of 100-200 nm in the best cases (Varga et al., 2016). These works characterize the osteocyte network and LCN of mineralized bone tissue, and reveal some texture that is attributed to the collagen banding pattern (Peyrin et al., 2014). However, while a larger volume is obtained, even using a synchrotron source, voxel sizes ranged from 50 to $130 \mathrm{~nm}$ (Wittig et al., 2019) to $280 \mathrm{~nm}$ (Peyrin et al., 2012), double to a full order of magnitude larger than the $25 \mathrm{~nm}$ obtained herein. Conversely, the advancements in image processing for X-ray data are significantly more developed than the nascent field of PFIB, and reconstructed and denoised to reveal fine details of bone structure (Dong et al., 2014; Pacureanu et al., 2013, 2012). Future PFIB-SEM evaluation of bone structure and LCN should focus on further optimization of acquisition parameters as well image processing techniques to reveal the structural and biological linkage in bone. Ultimately, PFIB-SEM could advantageously provide accurate quantification of local regions of the LCN if coupled with other less resolved methods which provide a visualization of larger portions of the network. 


\section{Study Limitations.}

While this paper provides an exciting proof-of-concept for using PFIB-SEM serial sectioning to analyze bone, we are aware that there are some limitations to our study design. Firstly, we recognize that the demineralized and mineralized datasets represent a single data point and from different orientations in bone - along and transverse to the long axis of the femur. Nevertheless, the findings presented in a single dataset are representative of volumes larger than several datasets of traditional FIB-SEM. Of course, our future work will probe a wider range of samples, both anatomical location and species. We do note that specimens were prepared by dehydration and embedding, rather than cryogenic FIB-SEM. Future possibilities for cryo-PFIB-SEM exist. Nevertheless, our findings are in line with structures shown in cryogenic-FIB-SEM, and embedded FIB-SEM published by others (Table 1). The volumes probed in this study, while comparable to the largest FIB-SEM datasets on bone, are still on the small scale for what is possible with PFIB-SEM. Larger volumes, capturing several osteocytes and their connections, will indeed be interesting to probe in the future, bringing PFIB-SEM to the level of investigations carried out with X-ray techniques. Lastly, in our work, we've analyzed a skeletally mature bone specimen, though it is true that the age of the osteon and specific lamella probed is unknown. While we anticipate the prolate ellipsoid or marquise structure is not associated only with mineralization fronts, future work comparing primary and secondary osteons would be interesting to shed light on whether this is a permanent or transient structure in lamellar bone.

\section{Conclusion.}

PFIB-SEM is a promising technique to probe both the nano and microscale hierarchy of bone. Herein, we demonstrate that PFIB-SEM serial sectioning can achieve volumes of thousands of microns cubed, while maintaining resolution of collagen fibrils on demineralized bone. The large volume and fast milling capabilities of this technique enabled an approach to begin probing the LCN and mineralized fibrils simultaneously. Moreover, this work demonstrates that analyzing mineralized bone, as opposed to demineralized bone, is essential for making claims on collagen-mineral arrangement and bone hierarchical structure in general. As suggested by others in human bone (Reznikov et al., 2018), rat bone (Shah et al., 2020) and turkey tendon (Zou et al., 2019), we confirm that the mineral in cortical human bone takes on a prolate ellipsoidal shape. Our large scale imaging confirms that these clusters are indeed densely packed across lamellar bone and not isolated events. The central cross-section of these ellipsoids represents the "rosette" features described previously (Grandfield et al., 2018). It is therefore clear that the organization of mineral is not solely restricted to its interversus intrafibrillar arrangement with collagen, but depends on coordination across several collagen fibrils. We suggest that the presence of inter-fibrillar mineral is likely on the exterior of these clusters, while intrafibrillar mineral appears to be a possibility in the interior. Further work is needed to probe this hypothesis and investigate how this arrangement changes across osteons, and of course, in other types of bone.

\section{Acknowledgements.}

We gratefully acknowledge Dr. Xiaoyue Wang for assistance with bone demineralization, staining and preliminary imaging. Sample preparation was conducted at the Faculty of Health Sciences Electron Microscopy Facility at McMaster University, with assistance by Marcia Reid. Electron microscopy was performed at the Canadian Centre for Electron Microscopy, a facility supported by NSERC and other governmental agencies. We graciously thank Ariana Hurley for aiding in the quantification of 3D mineral clusters. We extend our gratitude to Dr. Natalie Reznikov at Object Research Systems (ORS, Montreal) for Dragonfly software support for data visualization and processing of the demineralized dataset.

\section{Funding Sources.}

This work was supported by the Natural Sciences and Engineering Research Council of Canada (NSERC) (RGPIN-2014-06053), the France-Canada Research Fund (FCRF-2018-Grandfield), and the Ontario Ministry of Research, Science and Innovation (Early Researcher Award ER17-13-081). DMB and JD are supported by an NSERC CGS-D and NSERC PGS-D scholarship, respectively. 


\section{Author contributions.}

Conceptualization; DMB, AG, KG

Methodology: HY, DMB

Data curation; DMB, JD, HY

Formal analysis; DMB, JD

Funding acquisition and supervision; $\mathrm{AG}, \mathrm{KG}$

Writing: original draft; $\mathrm{DMB}, \mathrm{AG}, \mathrm{KG}$

Writing: review \& editing; DMB, JD, HY, AG, KG

\section{References.}

Addison, W.N., Nelea, V., Chicatun, F., Chien, Y.-C., Tran-Khanh, N., Buschmann, M.D., Nazhat, S.N., Kaartinen, M.T., Vali, H., Tecklenburg, M.M., Franceschi, R.T., McKee, M.D., 2014. Extracellular matrix mineralization in murine MC3T3-E1 osteoblast cultures: an ultrastructural, compositional and comparative analysis with mouse bone. Bone 71, 244-56. https://doi.org/10.1016/j.bone.2014.11.003

Akiva, A., Nelkenbaum, O., Schertel, A., Yaniv, K., Weiner, S., Addadi, L., 2019. Intercellular Pathways from the Vasculature to the Forming Bone in the Zebrafish Larval Caudal Fin: Possible Role in Bone Formation. J Struct Biol 206, 139-148. https://doi.org/10.1016/j.jsb.2019.02.011

Altmann, F., Young, R.J., 2014. Site-specific metrology, inspection, and failure analysis of three-dimensional interconnects using focused ion beam technology. J Micro Nanolithography Mems Moems 13, 011202-011202. https://doi.org/10.1117/1.jmm.13.1.011202

Bassim, N., Scott, K., Giannuzzi, L.A., 2014. Recent advances in focused ion beam technology and applications. MRS Bulletin 39, 317-325. https://doi.org/10.1557/mrs.2014.52

Bernard, G.W., Pease, D.C., 1969. An electron microscopic study of initial intramembranous osteogenesis. Am J Anat 125 , 271-290. https://doi.org/10.1002/aja.1001250303

Bigi, A., Ripamonti, A., Koch, M.H.J., Roveri, N., 1988. Calcified turkey leg tendon as structural model for bone mineralization. International Journal of Biological Macromolecules 10, 282-286-282-286.

Bonewald, L.F., 2011. The amazing osteocyte. J. Bone Miner. Res. 26, 229-238.

Bonucci, E., 1971. The Locus of Initial Calcification in Cartilage. Clin Orthop Relat R 78, 108-139. https://doi.org/10.1097/00003086-197107000-00010

Burnett, T.L., Kelley, R., Winiarski, B., Contreras, L., Daly, M., Gholinia, A., Burke, M.G., Withers, P.J., 2016. Large volume serial section tomography by Xe Plasma FIB dual beam microscopy. Ultramicroscopy 161, 119-129. https:// doi.org/10.1016/j.ultramic.2015.11.001

Ciani, C., Doty, S.B., Fritton, S.P., 2009. An effective histological staining process to visualize bone interstitial fluid space using confocal microscopy. Bone 44, 1015-1017.

Currey, J.D., 2002. Bones: structure and mechanics. Princeton University Press.

Dong, P., Haupert, S., Hesse, B., Langer, M., Gouttenoire, P.-J., Bousson, V., Peyrin, F., 2014. 3D osteocyte lacunar morphometric properties and distributions in human femoral cortical bone using synchrotron radiation micro-CT images. Bone 60, 172-185.

Genthial, R., Beaurepaire, E., Schanne-Klein, M.-C., Peyrin, F., Farlay, D., Olivier, C., Bala, Y., Boivin, G., Vial, J.-C., Débarre, D., Gourrier, A., 2017. Label-free imaging of bone multiscale porosity and interfaces using third-harmonic generation microscopy. Scientific Reports 7, 3419.

Georgiadis, M., Müller, R., Schneider, P., 2016. Techniques to assess bone ultrastructure organization: orientation and arrangement of mineralized collagen fibrils. J Roy Soc Interface 13, 20160088. https://doi.org/10.1098/rsif.2016.0088

Giraud-Guille, M.M., 1988. Twisted plywood architecture of collagen fibrils in human compact bone osteons. Calcif. Tissue Int. 42, 167-180.

Grandfield, K., Vuong, V., Schwarcz, H.P., 2018. Ultrastructure of Bone: Hierarchical Features from Nanometer to Micrometer Scale Revealed in Focused Ion Beam Sections in the TEM. Calcified Tissue Int 103, 606-616. https://doi.org/10.1007/s00223-018-0454-9 
Granke, M., Gourrier, A., Rupin, F., Raum, K., Peyrin, F., Burghammer, M., Saïed, A., Laugier, P., 2013. Microfibril Orientation Dominates the Microelastic Properties of Human Bone Tissue at the Lamellar Length Scale. Plos One 8, e58043. https://doi.org/10.1371/journal.pone.0058043

Hannah, K.M., Thomas, C.D.L., Clement, J.G., Carlo, F.D., Peele, A.G., 2010. Bimodal distribution of osteocyte lacunar size in the human femoral cortex as revealed by micro-CT. Bone 47, 866-871.

Hasegawa, T., Endo, T., Tsuchiya, E., Kudo, A., Shen, Z., Moritani, Y., Abe, M., Yamamoto, T., Hongo, H., Tsuboi, K., Yoshida, T., Nagai, T., Khadiza, N., Yokoyama, A., Freitas, P.H.L. de, Li, M., Amizuka, N., 2017. Biological application of focus ion beam-scanning electron microscopy (FIB-SEM) to the imaging of cartilaginous fibrils and osteoblastic cytoplasmic processes. J Oral Biosci 59, 55-62. https://doi.org/10.1016/j.job.2016.11.004

Hasegawa, T., Yamamoto, T., Hongo, H., Qiu, Z., Abe, M., Kanesaki, T., Tanaka, K., Endo, T., Freitas, P.H.L. de, Li, M., Amizuka, N., 2018. Three-dimensional ultrastructure of osteocytes assessed by focused ion beam-scanning electron microscopy (FIB-SEM). Histochem Cell Biol 149, 423-432. https://doi.org/10.1007/s00418-018-1645-1

Hashimoto, M., Nagaoka, N., Tabata, K., Tanaka, T., Osumi, R., Odagaki, N., Hara, T., Kamioka, H., 2017. Threedimensional morphometry of collagen fibrils in membranous bone. Integr Biology Quantitative Biosci Nano Macro 9, 868-875. https://doi.org/10.1039/c7ib00073a

Hirashima, S., Kanazawa, T., Ohta, K., Nakamura, K., 2020a. Three-dimensional ultrastructural imaging and quantitative analysis of the periodontal ligament. Anat Sci Int 95, 1-11. https://doi.org/10.1007/s12565-019-00502-5

Hirashima, S., Ohta, K., Kanazawa, T., Togo, A., Tsuneyoshi, R., Kusukawa, J., Nakamura, K., 2020b. Cellular network across cementum and periodontal ligament elucidated by FIB/SEM tomography. Microscopy 69, 53-58. https://doi.org/10.1093/jmicro/dfz117

Hu, C., Aindow, M., Wei, M., 2017. Focused ion beam sectioning studies of biomimetic hydroxyapatite coatings on Ti-6Al4V substrates. Surf Coatings Technology 313, 255-262. https://doi.org/10.1016/j.surfcoat.2017.01.103

Kamioka, H., Kameo, Y., Imai, Y., Bakker, A.D., Bacabac, R.G., Yamada, N., Takaoka, A., Yamashiro, T., Adachi, T., KleinNulend, J., 2012. Microscale fluid flow analysis in a human osteocyte canaliculus using a realistic high-resolution image-based three-dimensional model. Integr Biol 4, 1198-1206. https://doi.org/10.1039/c2ib20092a

Kanazawa, T., Gotoh, M., Ohta, K., Shiba, N., Nakamura, K.-I., 2014. Novel characteristics of normal supraspinatus insertion in rats: an ultrastructural analysis using three-dimensional reconstruction using focused ion beam/scanning electron microscope tomography. Muscles Ligaments Tendons 4, 182-7. https://doi.org/10.11138/mltj/2014.4.2.182

Kelley, R.D., Song, K., Leer, B.V., Wall, D., Kwakman, L., 2013. Xe+ FIB Milling and Measurement of Amorphous Silicon Damage. Microsc Microanal 19, 862-863. https://doi.org/10.1017/s1431927613006302

Kerschnitzki, M., Akiva, A., Shoham, A.B., Asscher, Y., Wagermaier, W., Fratzl, P., Addadi, L., Weiner, S., 2016a. Bone mineralization pathways during the rapid growth of embryonic chicken long bones. J Struct Biol 195, 82-92. https:// doi.org/10.1016/j.jsb.2016.04.011

Kerschnitzki, M., Akiva, A., Shoham, A.B., Koifman, N., Shimoni, E., Rechav, K., Arraf, A.A., Schultheiss, T.M., Talmon, Y., Zelzer, E., Weiner, S., Addadi, L., 2016b. Transport of membrane-bound mineral particles in blood vessels during chicken embryonic bone development. Bone 83, 65-72. https://doi.org/10.1016/j.bone.2015.10.009

Kerschnitzki, M., Wagermaier, W., Roschger, P., Seto, J., Shahar, R., Duda, G.N., Mundlos, S., Fratzl, P., 2011. The organization of the osteocyte network mirrors the extracellular matrix orientation in bone. J. Struct. Biol. 173, 303311.

Kollmannsberger, P., Kerschnitzki, M., Repp, F., Wagermaier, W., Weinkamer, R., Fratzl, P., 2017. The small world of osteocytes: connectomics of the lacuno-canalicular network in bone. New Journal of Physics 19, 073019-073019.

Landis, W.J., Hodgens, K.J., Arena, J., Song, M.J., McEwen, B.F., 1996. Structural relations between collagen and mineral in bone as determined by high voltage electron microscopic tomography. Microsc Res Techniq 33, 192-202.

Landis, W.J., Moradian-Oldak, J., Weiner, S., 1991. Topographic imaging of mineral and collagen in the calcifying Turkey tendon. Connective tissue research 25, 181-196.

Lee, B.E.J., Luo, L., Grandfield, K., Andrei, C.M., Schwarcz, H.P., 2019. Identification of collagen fibrils in cross sections of bone by electron energy loss spectroscopy (EELS). Micron 124, 102706. https://doi.org/10.1016/j.micron.2019.102706

Lin, Y., Xu, S., 2010. AFM analysis of the lacunar-canalicular network in demineralized compact bone. Journal of Microscopy 241, 291-302. 
Loeber, T.H., Laegel, B., Wolff, S., Schuff, S., Balle, F., Beck, T., Eifler, D., Fitschen, J.H., Steidl, G., 2017. Reducing curtaining effects in FIB/SEM applications by a goniometer stage and an image processing method. J Vac Sci Technology B Nanotechnol Microelectron Mater Process Meas Phenom 35, 06 GK01. https://doi.org/10.1116/1.4991638

Magal, R.A., Reznikov, N., Shahar, R., Weiner, S., 2014. Three-dimensional structure of minipig fibrolamellar bone: adaptation to axial loading. J Struct Biol 186, 253-64. https://doi.org/10.1016/j.jsb.2014.03.007

Maria, R., Ben-Zvi, Y., Rechav, K., Klein, E., Shahar, R., Weiner, S., 2019. An unusual disordered alveolar bone material in the upper furcation region of minipig mandibles: a 3D hierarchical structural study. J Struct Biol 206, 128-137. https://doi.org/10.1016/j.jsb.2019.02.010

McKee, M.D., Nanci, A., 1996. Osteopontin at mineralized tissue interfaces in bone, teeth, and osseointegrated implants: Ultrastructural distribution and implications for mineralized tissue formation, turnover, and repair. Microsc Res Techniq 33, 141-164. https://doi.org/10.1002/(sici)1097-0029(19960201)33:2<141::aid-jemt5>3.0.co;2-w

McNally, E.A., Schwarcz, H.P., Botton, G.A., Arsenault, A.L., 2012. A Model for the Ultrastructure of Bone Based on Electron Microscopy of Ion-Milled Sections. Plos One 7, e29258. https://doi.org/10.1371/journal.pone.0029258

Midura, R.J., Vasanji, A., Su, X., Midura, S.B., Gorski, J.P., 2008. Isolation of Calcospherulites from the Mineralization Front of Bone. Cells Tissues Organs 189, 75-79. https://doi.org/10.1159/000152914

Nudelman, F., Lausch, A.J., Sommerdijk, N.A.J.M., Sone, E.D., 2013. In vitro models of collagen biomineralization. J Struct Biol 183, 258-269. https://doi.org/10.1016/j.jsb.2013.04.003

Pacureanu, A., Langer, M., Boller, E., Tafforeau, P., Peyrin, F., 2012. Nanoscale imaging of the bone cell network with synchrotron X-ray tomography: optimization of acquisition setup. Med. Phys. 39, 2229.

Pacureanu, A., Larrue, A., Langer, M., Olivier, C., Muller, C., Lafage-Proust, M.-H., Peyrin, F., 2013. Adaptive filtering for enhancement of the osteocyte cell network in 3D microtomography images. IRBM 34, 48-52. https://doi.org/10.1016/j.irbm.2012.12.013

Peyrin, F., Dong, P., Pacureanu, A., Langer, M., 2014. Micro- and Nano-CT for the Study of Bone Ultrastructure. Curr Osteoporos Rep 12, 465-474. https://doi.org/10.1007/s11914-014-0233-0

Peyrin, F., Pacureanu, A., Zuluaga, M.A., Dong, P., Langer, M., 2012. 3D X-Ray CT imaging of the bone lacuno-canalicular network. 2012 9th IEEE Intl Symposium Biomedical Imaging (ISBI) 1, 1788-1791. https://doi.org/10.1109/isbi.2012.6235929

Repp, F., Kollmannsberger, P., Roschger, A., Kerschnitzki, M., Berzlanovich, A., Gruber, G.M., Roschger, P., Wagermaier, W., Weinkamer, R., 2017. Spatial heterogeneity in the canalicular density of the osteocyte network in human osteons. Bone Reports 6, 101-108.

Reznikov, N., Almany-Magal, R., Shahar, R., Weiner, S., 2013. Three-dimensional imaging of collagen fibril organization in rat circumferential lamellar bone using a dual beam electron microscope reveals ordered and disordered sublamellar structures. Bone 52, 676-683. https://doi.org/10.1016/j.bone.2012.10.034

Reznikov, N., Bilton, M., Lari, L., Stevens, M.M., Kröger, R., 2018. Fractal-like hierarchical organization of bone begins at the nanoscale. Science 360, eaao2189. https://doi.org/10.1126/science.aao2189

Reznikov, N., Chase, H., Brumfeld, V., Shahar, R., Weiner, S., 2014a. The 3D structure of the collagen fibril network in human trabecular bone: relation to trabecular organization. Bone 71, 189-95. https://doi.org/10.1016/j.bone.2014.10.017

Reznikov, N., Shahar, R., Weiner, S., 2014b. Three-dimensional structure of human lamellar bone: The presence of two different materials and new insights into the hierarchical organization. Bone 59, 93-104. https://doi.org/10.1016/j.bone.2013.10.023

Reznikov, N., Shahar, R., Weiner, S., 2014c. Bone hierarchical structure in three dimensions. Acta Biomater 10, 3815-3826. https://doi.org/10.1016/j.actbio.2014.05.024

Robles, H., Park, S., Joens, M.S., Fitzpatrick, J.A.J., Craft, C.S., Scheller, E.L., 2018. Characterization of the bone marrow adipocyte niche with three-dimensional electron microscopy. Bone 118, 89-98. https://doi.org/10.1016/j.bone.2018.01.020

Sano, H., Kikuta, J., Furuya, M., Kondo, N., Endo, N., Ishii, M., 2015. Intravital bone imaging by two-photon excitation microscopy to identify osteocytic osteolysis in vivo. Bone 74, 134-139.

Schneider, P., Meier, M., Wepf, R., Müller, R., 2011. Serial FIB/SEM imaging for quantitative 3D assessment of the osteocyte lacuno-canalicular network. Bone 49, 304-11. https://doi.org/10.1016/j.bone.2011.04.005 
Schwarcz, H.P., 2015. The ultrastructure of bone as revealed in electron microscopy of ion-milled sections. Semin Cell Dev Biol 46, 44-50. https://doi.org/10.1016/j.semcdb.2015.06.008

Shah, F.A., Palmquist, A., 2017. Evidence that Osteocytes in Autogenous Bone Fragments can Repair Disrupted Canalicular Networks and Connect with Osteocytes in de novo Formed Bone on the Fragment Surface. Calcified Tissue Int 101, 321-327. https://doi.org/10.1007/s00223-017-0283-2

Shah, F.A., Ruscsák, K., Palmquist, A., 2020. Transformation of bone mineral morphology: From discrete marquise-shaped motifs to a continuous interwoven mesh. Bone Reports 100283. https://doi.org/10.1016/j.bonr.2020.100283

Shah, F.A., Zanghellini, E., Matic, A., Thomsen, P., Palmquist, A., 2016. The Orientation of Nanoscale Apatite Platelets in Relation to Osteoblastic-Osteocyte Lacunae on Trabecular Bone Surface. Calcified Tissue Int 98, 193-205. https://doi.org/10.1007/s00223-015-0072-8

Silvent, J., Akiva, A., Brumfeld, V., Reznikov, N., Rechav, K., Yaniv, K., Addadi, L., Weiner, S., 2017. Zebrafish skeleton development: High resolution micro-CT and FIB-SEM block surface serial imaging for phenotype identification. Plos One 12, e0177731. https://doi.org/10.1371/journal.pone.0177731

Smith, N.S., Skoczylas, W.P., Kellogg, S.M., Kinion, D.E., Tesch, P.P., Sutherland, O., Aanesland, A., Boswell, R.W., 2006. High brightness inductively coupled plasma source for high current focused ion beam applications. J Vac Sci Technology B Microelectron Nanometer Struct 24, 2902. https://doi.org/10.1116/1.2366617

Tanoue, R., Ohta, K., Miyazono, Y., Iwanaga, J., Koba, A., Natori, T., Iwamoto, O., Nakamura, K., Kusukawa, J., 2018. Three-dimensional ultrastructural analysis of the interface between an implanted demineralised dentin matrix and the surrounding newly formed bone. Sci Reports 8, 2858. https://doi.org/10.1038/s41598-018-21291-3

Varga, P., Hesse, B., Langer, M., Schrof, S., Männicke, N., Suhonen, H., Pacureanu, A., Pahr, D., Peyrin, F., Raum, K., 2015. Synchrotron X-ray phase nano-tomography-based analysis of the lacunar-canalicular network morphology and its relation to the strains experienced by osteocytes in situ as predicted by case-specific finite element analysis. Biomechanics and Modeling in Mechanobiology 14, 267-282.

Varga, P., Weber, L., Hesse, B., Langer, M., 2016. X-ray and Neutron Techniques for Nanomaterials Characterization 1-42. https://doi.org/10.1007/978-3-662-48606-1_1

Wagermaier, W., Gupta, H.S., Gourrier, A., Burghammer, M., Roschger, P., Fratzl, P., 2006. Spiral twisting of fiber orientation inside bone lamellae. Biointerphases 1, 1-5.

Wang, X., Shah, F.A., Palmquist, A., Grandfield, K., 2016. 3D Characterization of Human Nano-osseointegration by OnAxis Electron Tomography without the Missing Wedge. Acs Biomater Sci Eng 3, 49-55. https://doi.org/10.1021/acsbiomaterials.6b00519

Weiner, S., Traub, W., 1992. Bone structure: from angstroms to microns. FASEB 6, 879-885. https://doi.org/10.1096/fasebj.6.3.1740237

Weiner, S., Traub, W., 1989. Crystal size and organization in bone. Connect Tissue Res 21, 259-65. https://doi.org/10.3109/03008208909050015

Weiner, S., Traub, W., Wagner, H.D., 1999. Lamellar bone: structure-function relations. J. Struct. Biol. 126, 241-255.

Wittig, N.K., Laugesen, M., Birkbak, M.E., Bach-Gansmo, F.L., Pacureanu, A., Bruns, S., Wendelboe, M.H., Brüel, A., Sørensen, H.O., Thomsen, J.S., Birkedal, H., 2019. Canalicular Junctions in the Osteocyte Lacuno-Canalicular Network of Cortical Bone. ACS Nano 13, 6421-6430. https://doi.org/10.1021/acsnano.8b08478

You, L., Weinbaum, S., Cowin, S.C., Schaffler, M.B., 2004. Ultrastructure of the osteocyte process and its pericellular matrix. Anatomical Rec Part Discov Mol Cell Evol Biology 278A, 505-513. https://doi.org/10.1002/ar.a.20050

Zou, Z., Tang, T., Macías-Sánchez, E., Sviben, S., Landis, W.J., Bertinetti, L., Fratzl, P., 2019. Three-dimensional Structural Interrelations between Cells, Extracellular Matrix and Mineral in Vertebrate Mineralization. BioRxiv 803007. https://doi.org/10.1101/803007 


\section{Supplementary information}

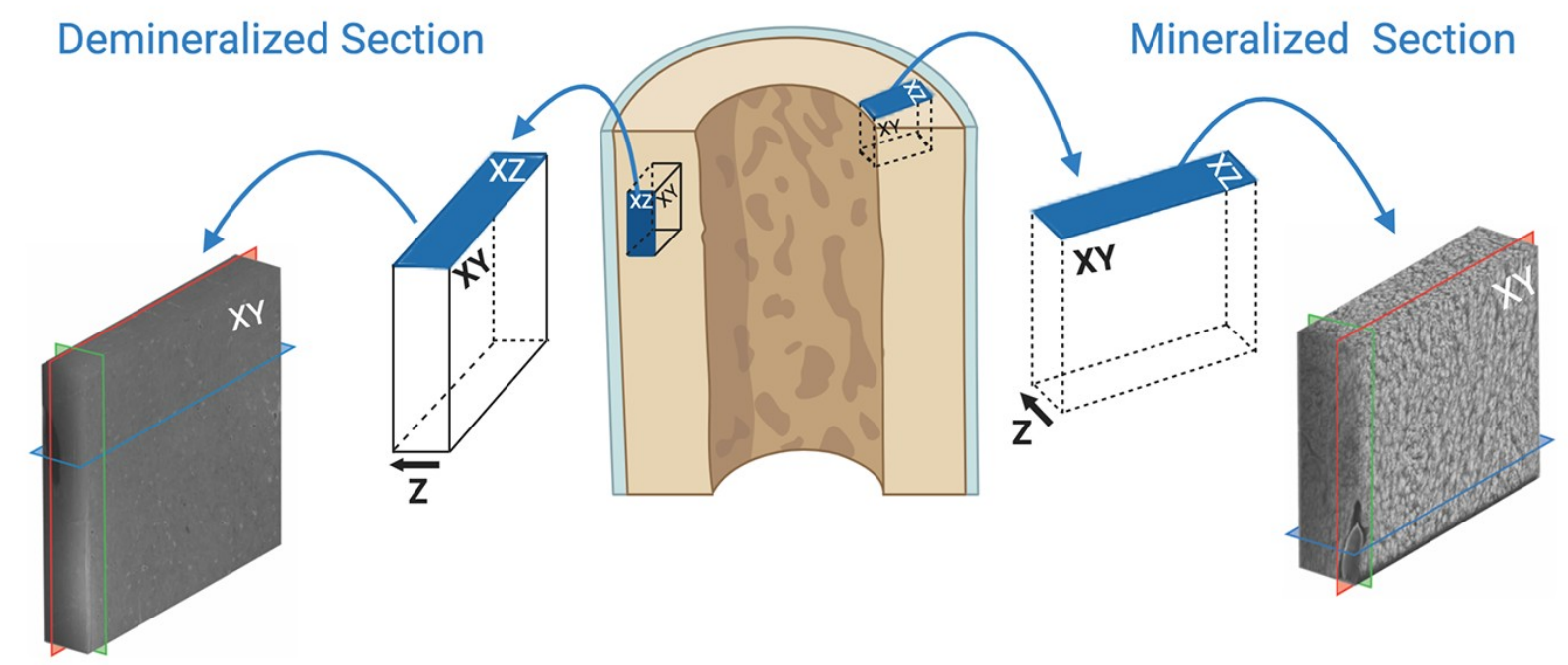

Figure S1. Schematic of sample orientations. The samples were originally cut in the longitudinal (demineralized) and transverse (mineralized) direction. Therefore, the blue planes highlighted above sit on cut surfaces, and represent the top surface viewed in the SEM. In PFIB-SEM, the XY plane is generally assigned normal to the top surface - it represents the surface that is sequentially milled and imaged. The $Z$ direction in PFIB-SEM thereby dictates the direction of sequential milling, and eventually the thickness of the volume.

Although the samples were oriented orthogonal to one another, we can see from above that their XY planes both contain the long axis of the bone. Corresponding final tomograms are on the far left and right. Segmented canaliculi in Figure 8 and 9 suggest that the demineralized XY plane was oriented radially with respect to an osteon, while the mineralized XY plane is likely tangential. Created with BioRender, www.biorender.com.

Methods for Capping Layer Composition Experiments: To determine a suitable protective layer against ion beam damage for demineralized and mineralized bone tissue, due to their varying hardness, a variety of protective layer compositions were deposited on the specimens. We explored several options, non-systematically. In the gas injection system, the pure Pt and $\mathrm{W}$ deposits are approximately $20 \% \mathrm{C}$ due to the gas mixture in the gas injection system (Utke et al., 2008). For our purposes, we refer to this as 'pure' in Table S1 to avoid confusion. Along with the pure compositions, various flux percentages (\%), given as a fraction of the maximum gas output for that element, were explored. After deposition, slices of material were removed using the beam conditions given in Table S2 and the quality of cross-section was assessed qualitatively. A "good" capping layer was determined to have minimal curtaining artifacts by qualitative assessment (Table S1). Our findings (Figure S2 and Table S1) indicate that demineralized bone sliced with a pure Pt capping layer produced the fewest artifacts, while mineralized bone produced the best quality when protected with pure $\mathrm{C}$. These results make sense considering the relative hardness of the capping layers and underlying material, matching a softer Pt coating to demineralized bone, and a harder $\mathrm{C}$ coating to mineralized bone. Of course, a larger, more systematic study would be interesting, as most FIB-SEM tomography is conducted with a standard Pt coating. 
Table S1: Various capping layers were non-systematically investigated to determine optimum composition for minimizing curtaining on demineralized and mineralized bone samples during PFIB-SEM tomography acquisition. The quality of the cross-section, given by a reduction in curtaining artifacts, is ranked qualitatively.

\begin{tabular}{lccccc}
\hline & & \multicolumn{3}{c}{ Capping Layer Composition } & \\
\hline $\begin{array}{l}\text { Quality of Cross } \\
\text { Section }\end{array}$ & Pure C & Pure W & Pure Pt & $\begin{array}{c}80 \% \mathrm{C} \text { to } \\
100 \% \mathrm{Pt}\end{array}$ & $90 \% \mathrm{C}$ to $100 \% \mathrm{Pt}$ \\
$\begin{array}{l}\text { Demineralized } \\
\begin{array}{l}\text { Bone } \\
\text { Mineralized Bone }\end{array}\end{array}$ & Fair & Good & Good & N/A & N/A \\
\hline
\end{tabular}

Table S2: Electron and ion beam parameters for cross-sectional milling of demineralized and mineralized bone during protective layer composition experiments. An in-lens detector in backscattered mode was used.

\begin{tabular}{lcc}
\hline & Demineralized Bone & Mineralized Bone \\
\hline Protective layer size & $10 \times 20 \times 5 \mu \mathrm{m}$ & $20 \times 40 \times 5 \mu \mathrm{m}$ \\
Electron beam & $1 \mathrm{keV}, 1.6 \mathrm{nA}$ & $1 \mathrm{keV}, 3.2 \mathrm{nA}$ \\
Working distance & $4.1 \mathrm{~mm}$ & $4.1 \mathrm{~mm}$ \\
lon beam & $1 \mu \mathrm{A}, 30 \mathrm{keV}$ & $0.5 \mu \mathrm{A}, 30 \mathrm{keV}$ \\
Slice thickness & $15 \mathrm{~nm}$ & $100 \mathrm{~nm}$ \\
Number of slices & 30 & 5 \\
Rocking angle & 4 & 0 \\
\hline
\end{tabular}
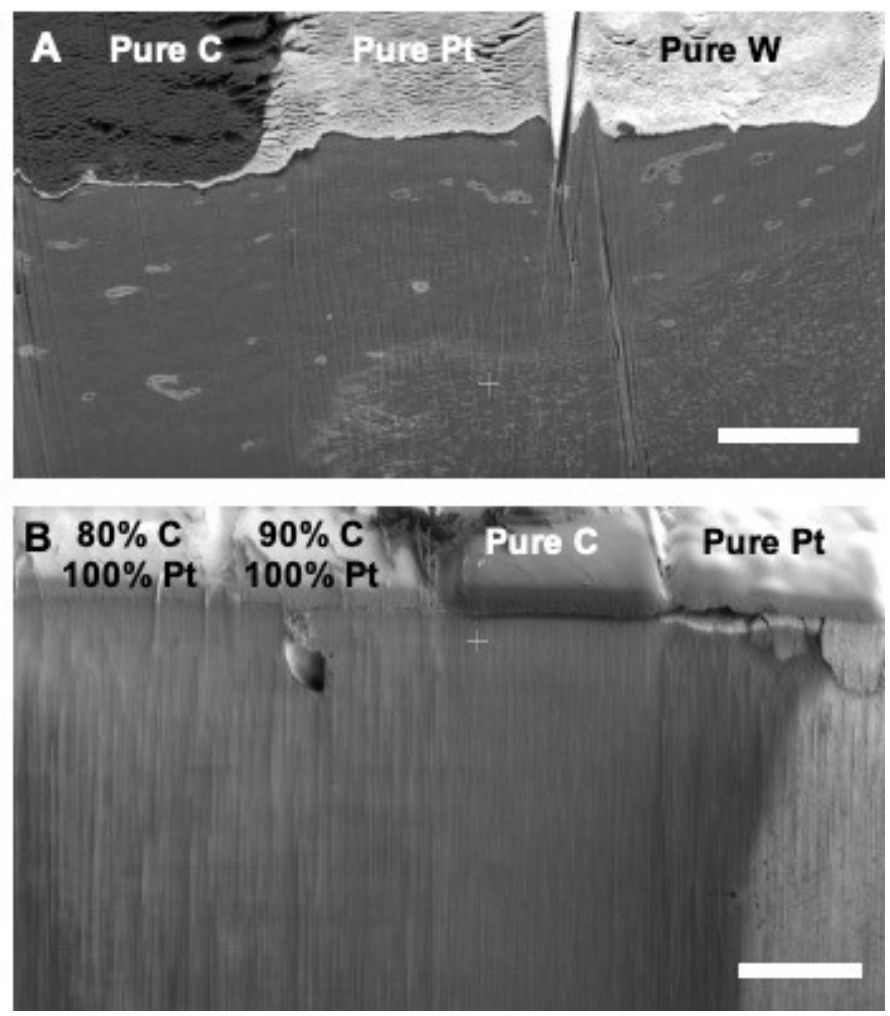

Figure S2. Capping layer composition experiments on demineralized and mineralized bone. (A) Deposition of C, $\mathrm{Pt}$, and $\mathrm{W}$ on demineralized bone after a small 30 slice tomography acquisition. A reduction in curtaining was noted under the Pt and W deposition layers. Scale bar: $5 \mu \mathrm{m}$. (B) Deposition of $\mathrm{C}, \mathrm{Pt}$, and $\mathrm{C} / \mathrm{Pt}$ mixtures on mineralized bone after a 5 slice tomography acquisition. A clear reduction in curtaining was observed under the $\mathrm{C}$ deposition layer. Percentages refer to the flux rate of gas relative to the standard deposition flux rate in the PFIB. Scale bar: $20 \mu \mathrm{m}$. 

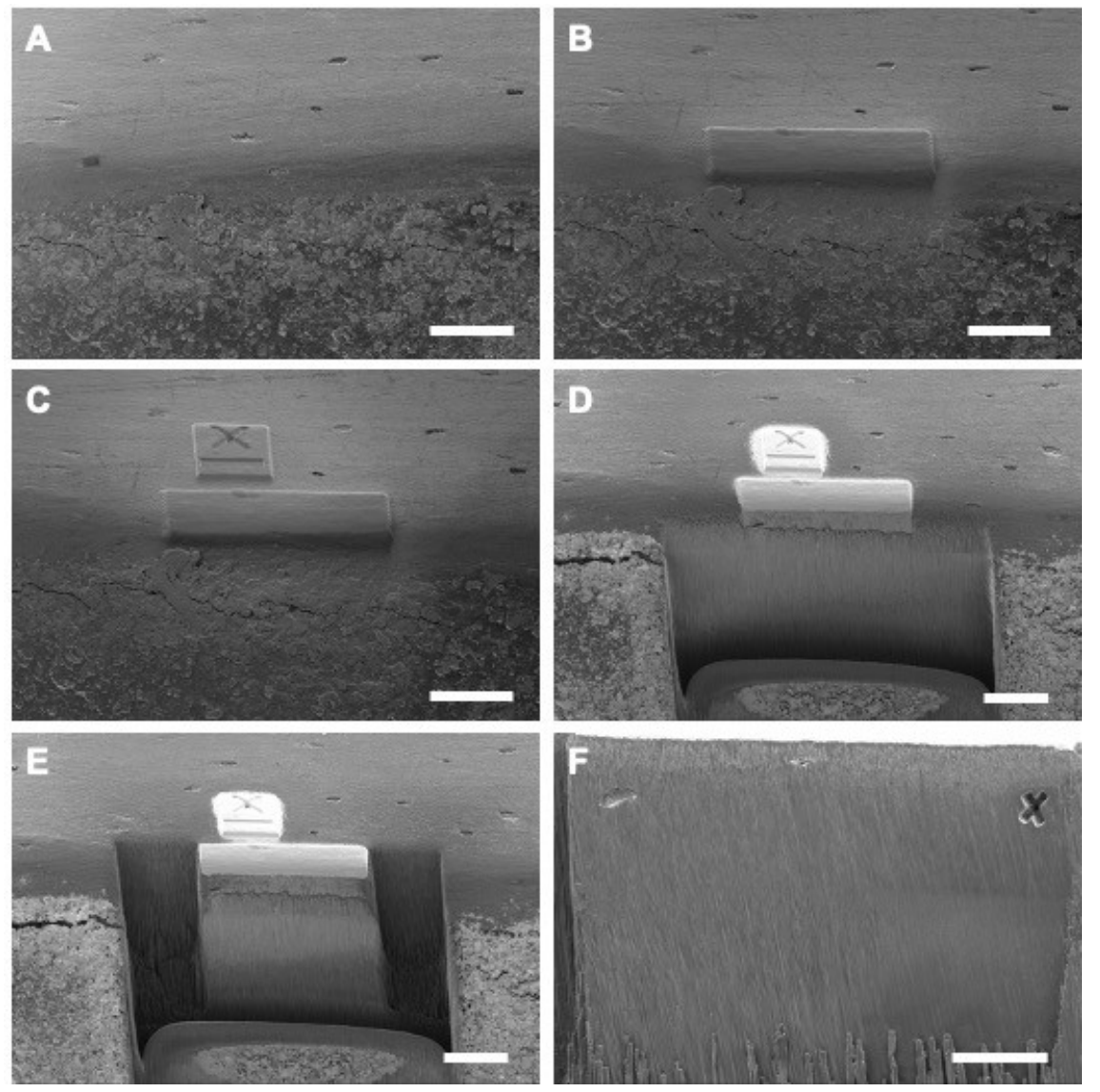

Figure S3. PFIB-SEM serial sectioning process. (A) A region of interest is selected. (B) The optimal protective capping layer is deposited on the region of interest, in this case, this is on the surface of the transverse plane. (C) A FIB fiducial is deposited and milled such that area can be tracked during automated serial sectioning. (D) A large cross section is exposed, this represents the XY milling and image plane. (E) Side trenches are milled to prevent shadowing and re-deposition. (F) A SEM fiducial is milled onto the cross-section to allow for easier image post-processing. All scale bars are $25 \mu \mathrm{m}$. 

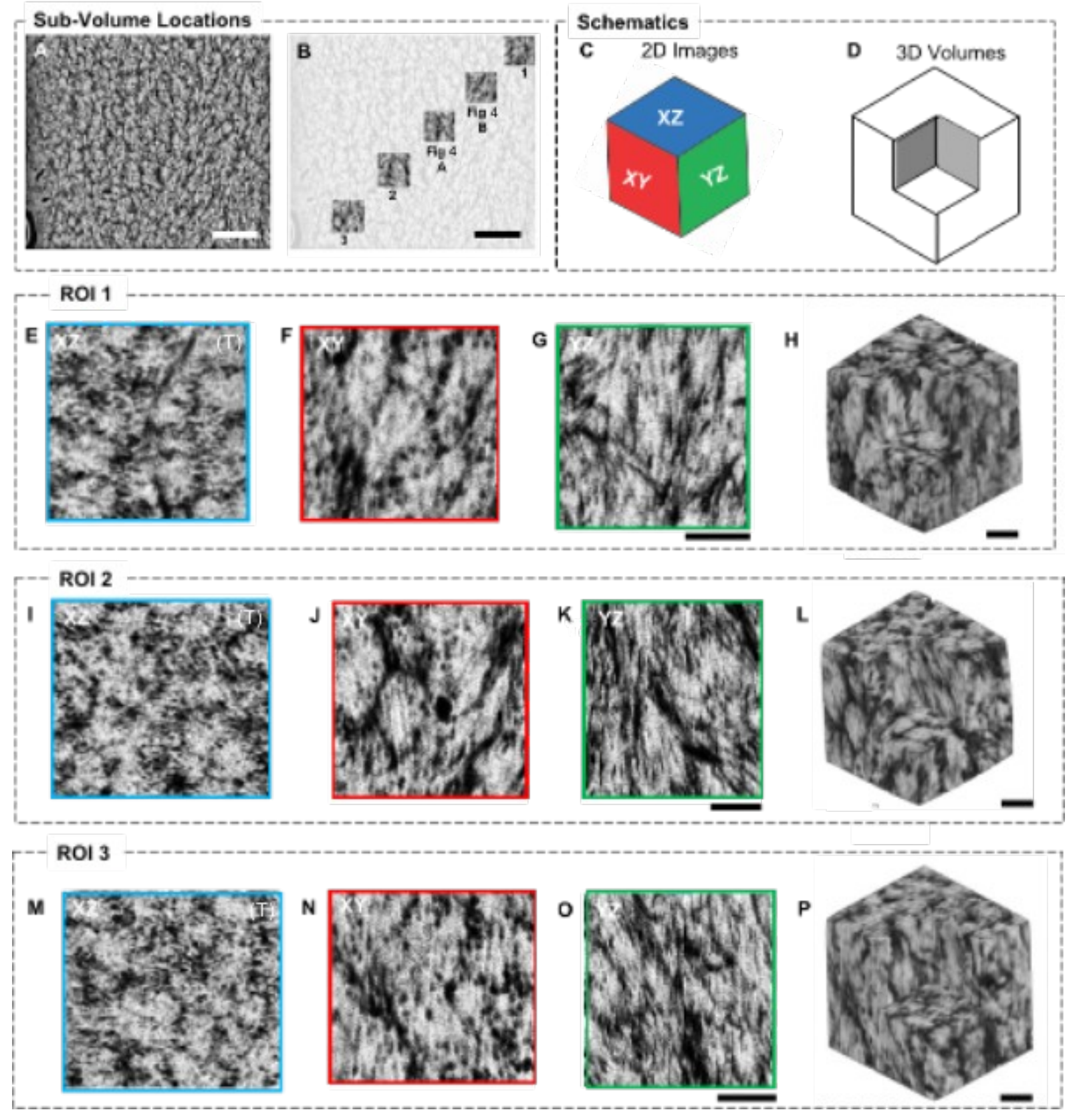

Figure S4. Exploring the mineral cluster orientation in 3D. Several additional ROIs (ROI 1,2,3) to the main manuscript Figures $6 \mathrm{~A}$ and $\mathrm{B}$ are included here across the mineralized dataset. Consistent orientation of the mineralized features is noted, with rosettes appearing in the XZ (or transverse to the long axis of the femur), while collagen banding is regularly visible in the $\mathrm{YZ}$ plane along the length of the femur. The marquise-, or diamond-shaped motif, which makes up the ellipsoid in 3D space is consistently visible throughout the $\mathrm{XY}$ and YZ planes. ROIs measure 3 × 3 × $3 \mu \mathrm{m} 3$. Scale bar: $1 \mu \mathrm{m}$. 

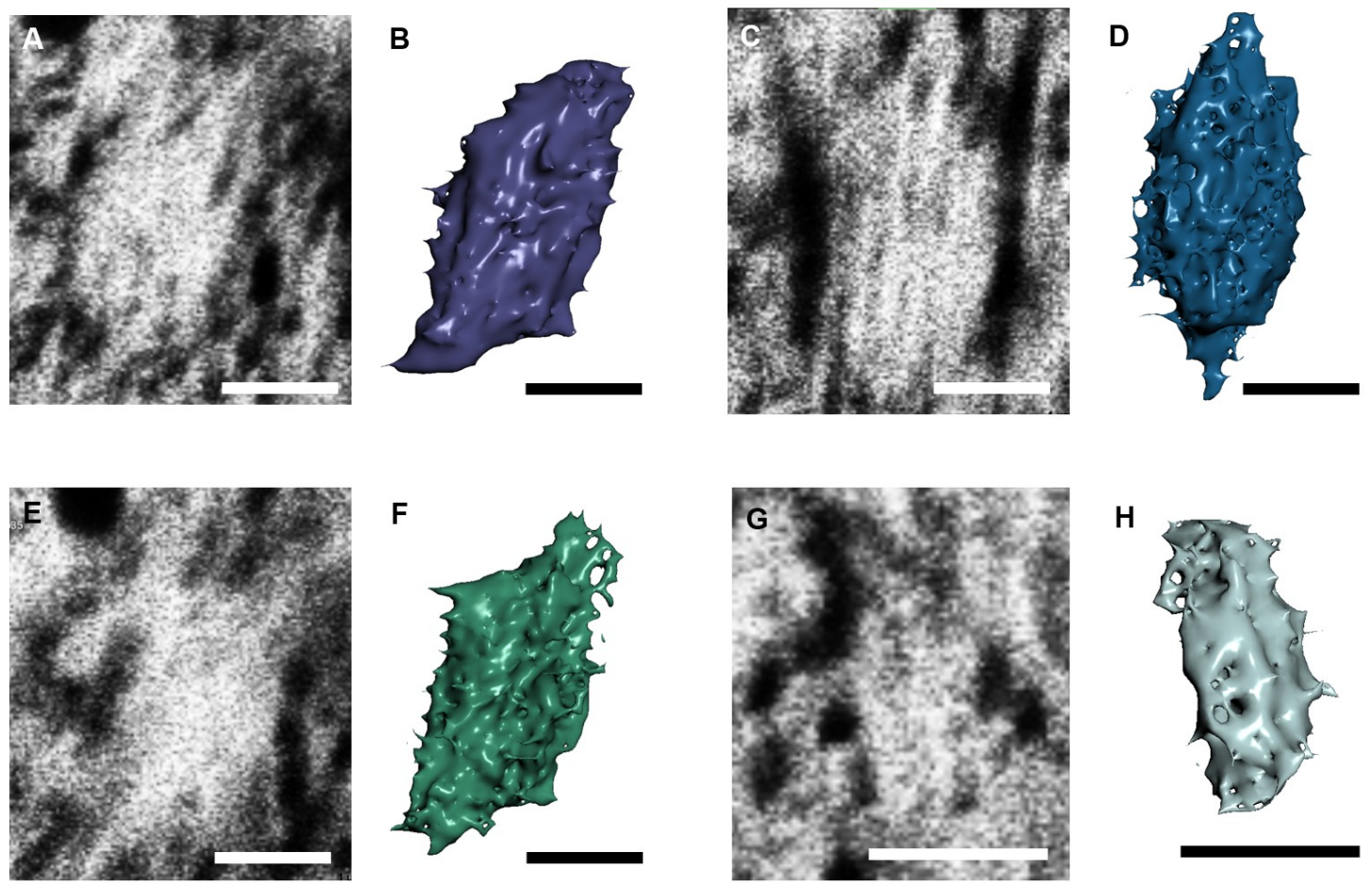

Figure S5: Segmented Prolate Ellipsoids. Serval 2D PFIB-SEM images of mineral marquise shapes and corresponding 3D reconstructed prolate ellipsoids at the same magnification. Discrete prolate spheroids were identified throughout the entire mineralized tomogram, only four are shown here. Scale bars: $500 \mathrm{~nm}$.

Methods Prolate Ellipsoid Segmentation: Mineral clusters were individually segmented in 3D. Grayscale histograms were split using Otsu's method in available ORS Dragonfly on the XZ plane of the mineralized dataset, keeping higher grayscale values to reveal 2D rosette structures. Discrete rosettes in each XZ slice were then manually segmented from the resulting region to reveal the 3Dshape of the mineral clusters. The maximum length and mid-point diameter of each 3D mineral shape was measured for 8 segmented volumes. Final segmentations are presented as smoothed meshes, after which maximum tip-to-tail length and diameter at midpoint were measured.

Table S3: Quantification of eight random prolate ellipsoids segmented from mineralized bone, where Avg is the average and S.D. is the standard deviation.

$\begin{array}{cc}\begin{array}{c}\text { Maximum } \\ \text { Length }(\mathrm{nm}) \\ 1138\end{array} & \begin{array}{c}\text { Maximum } \\ \text { Diameter }(\mathrm{nm})\end{array} \\ 1125 & 875 \\ 1250 & 612 \\ 1575 & 525 \\ 962 & 788 \\ 1388 & 875 \\ 862 & 825 \\ 825 & 638 \\ 1140 \mathrm{~nm} & 450 \\ 260 \mathrm{~nm} & 700 \mathrm{~nm} \\ \end{array}$



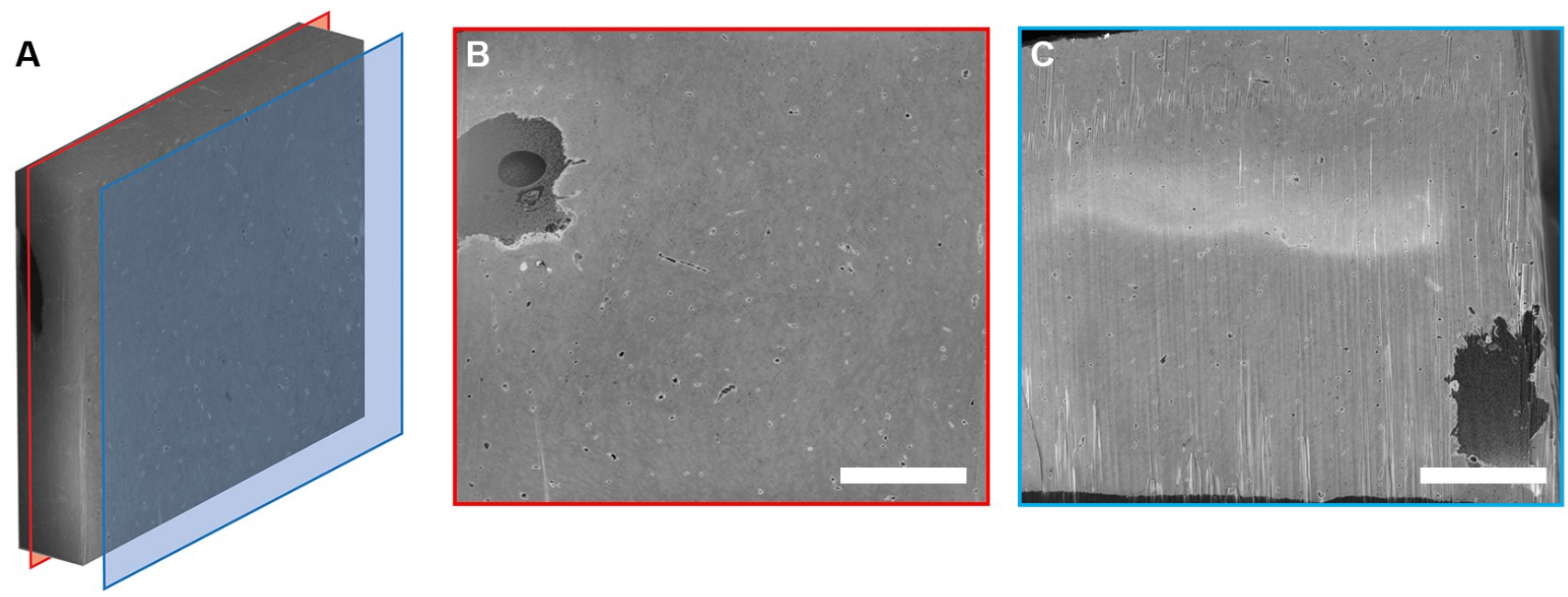

Figure S6. Two osteocytes present in demineralized bone. The demineralized dataset represents canalicular networks from two adjacent cells. The first osteocyte, bottom right of the blue plane, was encountered during preliminary milling. As PFIB serial sectioning requires a fine surface polish, several of the first slices, including this blue plane, are outside of the final volume recorded in the Slice and View tomogram. The second osteocyte, the top left of the red plane, is the osteocyte featured in the main manuscript. This juxtaposition of the two cells may account for the higher canalicular density observed in the demineralized dataset. Scale bar: $5 \mu \mathrm{m}$.

\section{Demineralized}

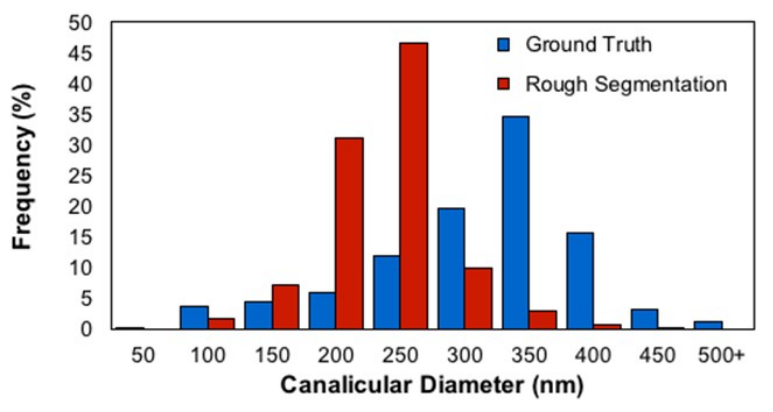

\section{Mineralized}

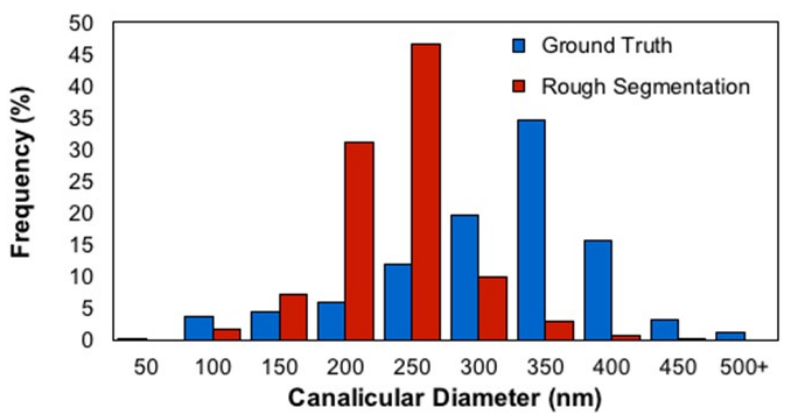

Figure S7. Dependence on neural network training quality. Results of two separate training inputs for (A) Demineralized tissue (B) Mineralized tissue. In the ground truth segmentations, training slices were diligently prepared where canalicular edges and interiors were included with only a small margin of error in guided segmentation. Rough segmentations included omissions of canaliculi or incomplete identification within single canaliculi, resulting in an apparent downward shift in the average canalicular diameter. A sufficient number of epochs were run in all cases to cause weighting factor convergence. 


\section{Movie captions.}

Movie S1. Orthogonal slices down the XZ plane of the mineralized dataset show the clear rosette pattern through the entire section with canaliculi (black) extending throughout.

Movie S2. The morphology of the mineral clusters. Orthogonal slices of marquise or ellipsoidal shapes in mineralized bone. An isolated mineral marquise (white) shown with orthogonal slices in the $\mathrm{XY}$ and $\mathrm{XZ}$ planes while rotating. At 10 seconds, the top-down view is shown, with the shape of a rosette apparent.

Movie S3. 2D slice-by-slice of rosettes. Single reconstructed slices from the XZ plane showing a zoomed in rosette shape as visualized in PFIB-SEM.

Movie S4. Orthogonal slices and LCN volume rendering of the demineralized dataset. From $0-0: 30$ seconds, demineralized bone tomogram rotates $360^{\circ}$. 0:30-1:00 SEM images slice one-by-one in the XY plane from the front of the volume to the back. 1:00 - 1:30 SEM images slice one by one in the XY plane from the back of the volume to the front. 1:30 - 2:00 Tomogram disappears and the reconstructed LCN appear and rotate 360 ${ }^{\circ} .2: 00-2: 30$ Tomogram appears with the $\mathrm{LCN}$ and rotates $360^{\circ}$.

Movie S5. Orthogonal slices and LCN volume rendering of the mineralized dataset. From $0-0: 30$ seconds, mineralized bone tomogram rotates $360^{\circ}$. 0:30-1:00 SEM images slice one by one in the XY plane from the front of the volume to the back. 1:00 - 1:30 SEM images slice one by one in the XY plane from the back of the volume to the front. 1:30 - 2:00 Tomogram disappears and the reconstructed LCN appear and rotate 360 ${ }^{\circ}$. 2:00 - 2:30 Tomogram appears with the $\mathrm{LCN}$ and rotates $360^{\circ}$.

\section{References.}

Utke, I., Hoffmann, P., Melngailis, J., 2008. Gas-assisted focused electron beam and ion beam processing and fabrication. J Vac Sci Technology B Microelectron Nanometer Struct 26, 1197. https://doi.org/10.1116/1.2955728 